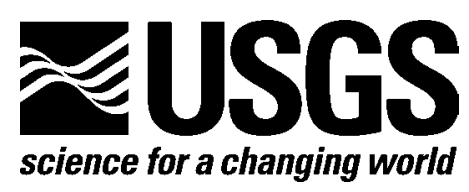

\title{
Guidelines for Standardized Testing of Broadband Seismometers and Accelerometers
}

By Charles R. Hutt, John R. Evans, Fred Followill, Robert L. Nigbor, and Erhard Wielandt

Open-File Report 2009-1295

U.S. Department of the Interior

U.S. Geological Survey 


\section{U.S. Department of the Interior KEN SALAZAR, Secretary \\ U.S. Geological Survey Marcia K. McNutt, Director}

U.S. Geological Survey, Reston, Virginia: 2010

For product and ordering information:

World Wide Web: http://www.usgs.gov/pubprod

Telephone: 1-888-ASK-USGS

For more information on the USGS - the Federal source for science about the Earth, its natural and living resources, natural hazards, and the environment:

World Wide Web: http://www.usgs.gov

Telephone: 1-888-ASK-USGS

Suggested citation:

Hutt, Charles R., Evans, John R., Followill, Fred, Nigbor, Robert L., and Wielandt, Erhard, 2010, Guidelines for standardized testing of broadband seismometers and accelerometers: U.S. Geological Survey Open-File Report, 2009-1295, 62 p.

Any use of trade, product, or firm names is for descriptive purposes only and does not imply endorsement by the U.S. Government.

Although this report is in the public domain, permission must be secured from the individual copyright owners to reproduce any copyrighted material contained within this report. 


\section{Contents}

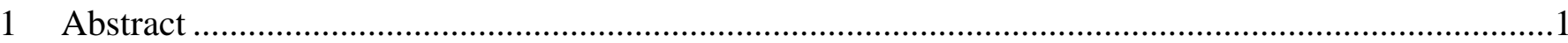

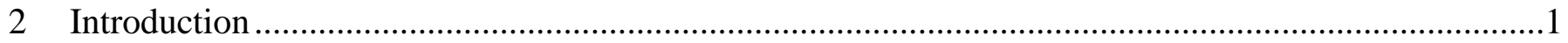

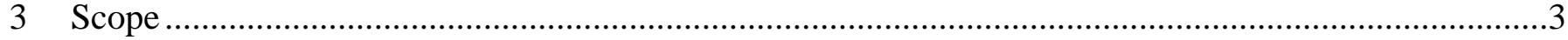

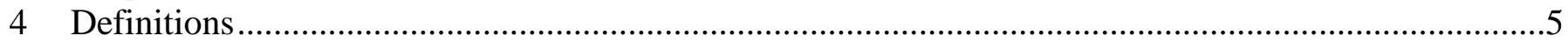

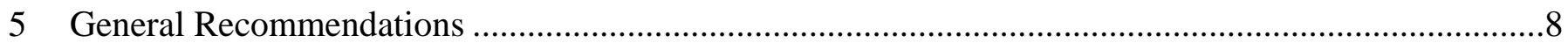

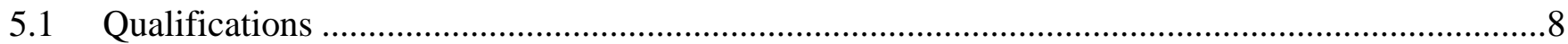

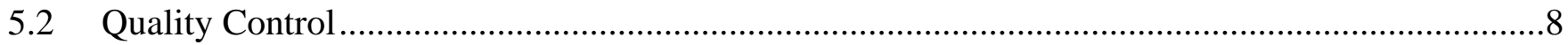

5.3 Test Equipment Calibration..............................................................................................

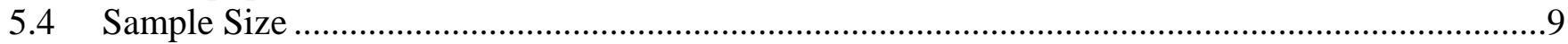

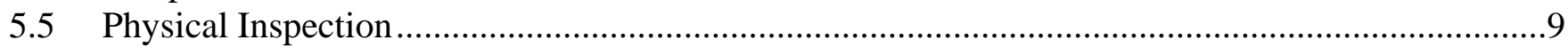

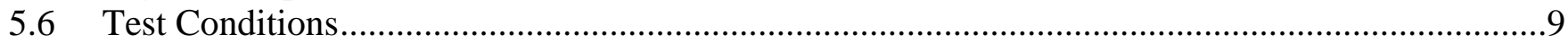

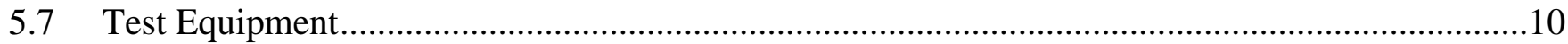

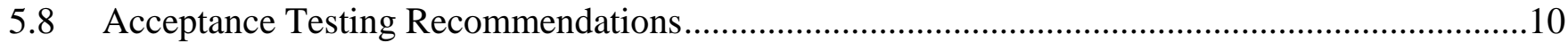

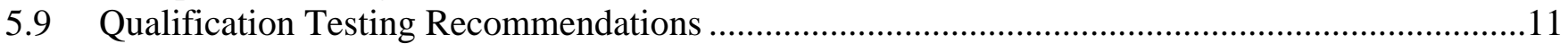

6 Recommended Testing for Weak Motion Broadband Velocity Sensors ..............................................12

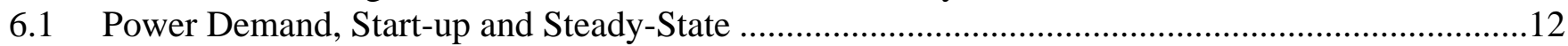

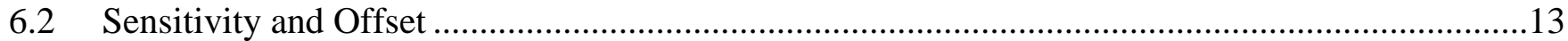

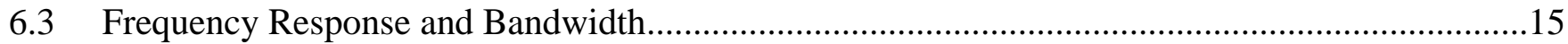

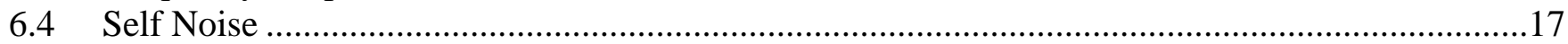

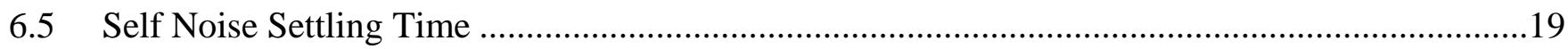

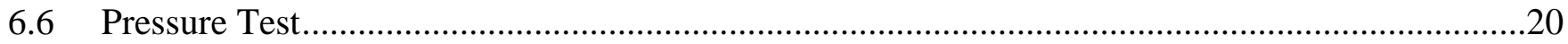

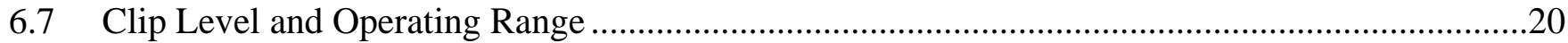

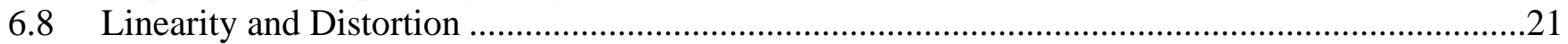

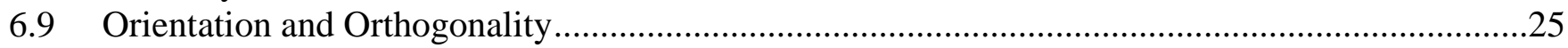

6.10 Temperature Effect on Sensitivity, Offset, and Mass Position .......................................................25

6.11 Power Supply Voltage Effect on Sensitivity ................................................................................26

6.12 Power Supply Noise and Voltage Effects on Seismometer Noise ................................................27

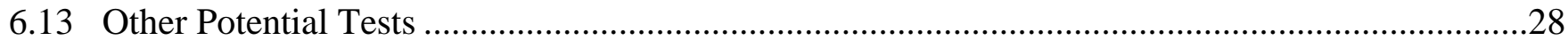

7 Recommended Testing for Strong Motion Acceleration Sensors...............................................................30

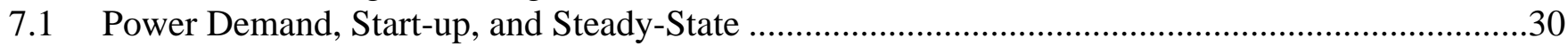

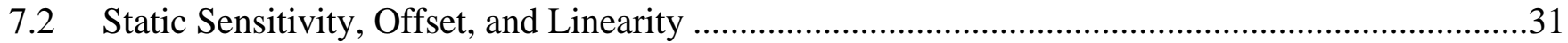

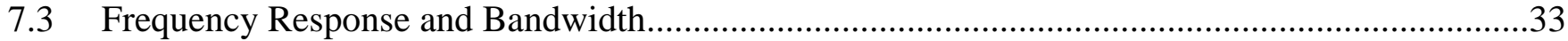

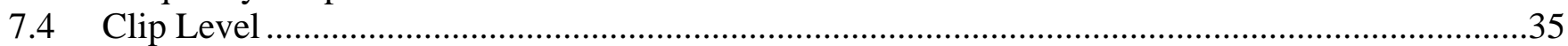

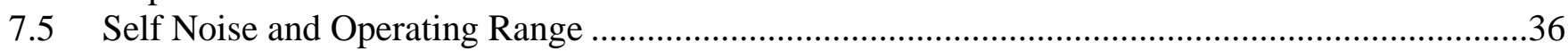

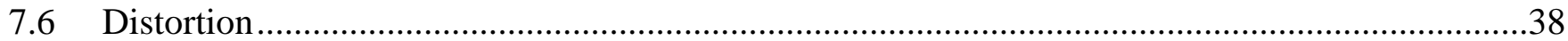

7.7 Orientation (Case to Actual) and Orthogonality ………...............................................................39

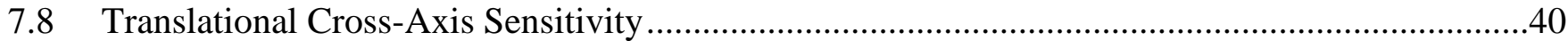

7.9 Temperature Effect on Sensitivity and Offset..............................................................................41

7.10 Power Supply Voltage and Voltage-Noise Effects on Offset and Sensitivity.................................42

7.11 Double Integration of Band-Limited Displacement Square Wave....................................................43

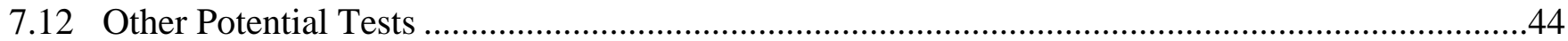

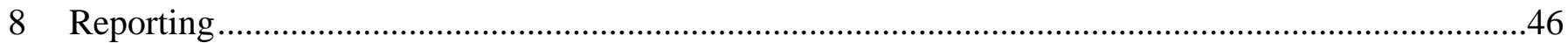

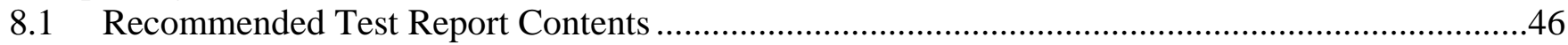

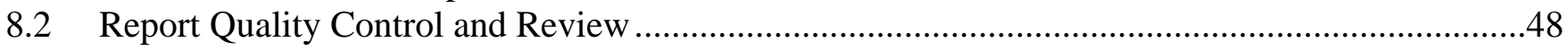




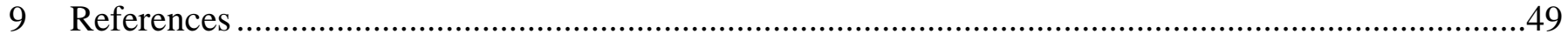

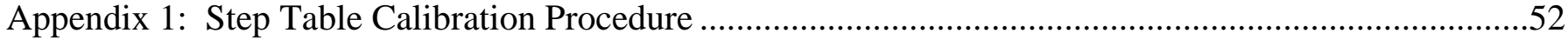

Appendix 2: Sample Test Procedure for WM BB Velocity Sensor Frequency Response 


\title{
Guidelines for Standardized Testing of Broadband Seismometers and Accelerometers
}

\author{
Charles R. Hutt ${ }^{1}$, John R. Evans ${ }^{2}$, Fred Followill ${ }^{3}$, Robert L. Nigbor ${ }^{4}$, and Erhard Wielandt ${ }^{5}$
}

\section{Abstract}

Testing and specification of seismic and earthquake-engineering sensors and recorders has been marked by significant variations in procedures and selected parameters. These variations cause difficulty in comparing such specifications and test results.

In July 1989, and again in May 2005, the U.S. Geological Survey hosted international public/private workshops with the goal of defining widely accepted guidelines for the testing of seismological inertial sensors, seismometers, and accelerometers. The Proceedings of the 2005 workshop have been published and include as appendix 6 the report of the 1989 workshop.

This document represents a collation and rationalization of a single set of formal guidelines for testing and specifying broadband seismometers and accelerometers.

\section{Introduction}

These guidelines for broadband seismometer and accelerometer testing were developed on the basis of recommendations of the Second Guidelines for Seismometer Testing Workshop (GST2) that was held in Albuquerque in May, 2005. The participants in GST2 included 42 seismic instrumentation specialists, including users from research, government, and treatymonitoring organizations; and 13 representatives from eight seismological-equipment manufacturers. Participants formed four Working Groups that provided recommendations on specific aspects of seismometer testing and reporting. These recommendations are documented in the GST2 proceedings (Hutt and others, 2009). The overall recommendation of the workshop, quoting from the GST2 Proceedings was:

"We recommend that a much smaller group, perhaps three to five individuals, be formed to draft these formal Guidelines for Seismometer Testing, based on the GST2 working group reports, ANSS instrumentation guidelines, and the earlier work of GST1.” (Hutt and others, 2009, page 4)

\footnotetext{
${ }^{1}$ U.S. Geological Survey, Albuquerque, N. Mex.

${ }^{2}$ U.S. Geological Survey, Menlo Park, Cali.

${ }^{3}$ Consultant, Livermore, Cali.

${ }^{4}$ University of California at Los Angeles, Los Angeles, Cali.

${ }^{5}$ Stuttgart University, Stuttgart, Germany
} 
This guidelines document was drafted by the authors in response to the GST2 recommendation. In addition to internal United States Geological Survey (USGS) review, a final draft was circulated to the GST2 participants and to other selected members of the seismology community for review and comment. Comments received were incorporated in this version. 


\section{Scope}

This document provides guidance for standardized performance testing of weak-motion broadband seismometers and strong-motion accelerometers used in all scales of seismic monitoring, from local to global. Applications of these guidelines include:

- Acceptance Testing by purchasers, either pre-award or post-award, to confirm required performance;

- Qualification Testing by vendors in response to contractual requirements or other criteria; and

- Exploratory Testing by seismometer users or developers, including all applications not covered under Acceptance or Qualification Testing.

The USGS and the United States Advanced National Seismic System (ANSS) plan to use these guidelines for purposes of testing and characterizing broadband (BB) seismometers, including weak motion (WM) BB velocity sensors and strong motion (SM) acceleration sensors. Guideline applications will include testing in support of instrument procurement (pre-award and post-award Acceptance Testing) and performance monitoring.

The scope of these guidelines includes performance testing of three-component WM BB velocity sensors and SM acceleration sensors, which are the two sensing components of many broadband seismic stations in networks such as the Global Seismic Network (GSN) and the ANSS. The scope does not include short-period passive seismometers and geophones, as performance testing of this common class of seismic sensors is fairly well covered within the research and exploration geophysics communities. The scope also does not include less common seismic sensors such as SM velocity seismometers, rotational seismometers, GPS systems, pore water pressure sensors, or strain meters.

While this document provides test procedures, they are often generic in nature and intended to be widely applicable. No attempt is made herein to provide specific criteria, limits, or other thresholds for individual sensors. These are application-specific and must be provided elsewhere for specific uses or procurements.

Table 1 lists 11 performance measurements for which test procedures are provided in this document. In Table 1, the tests are denoted Primary (P) or Secondary (S) for the two classes of sensors. Although all 11 tests are considered important for characterization of seismometer performance, the Primary tests are considered basic for Acceptance Testing purposes.

Detailed test procedures, each with Purpose, Test Equipment, Setup and Procedure, Analysis, and Results sections, are provided in section 6 for WM BB velocity sensors and section 7 for SM acceleration sensors. Sections 6.13 and 7.12 list other potential performance tests that may be considered, but no details are provided for these tertiary tests. The Test Procedures are preceded by discussions of Definitions (section 4), and General Recommendations (section 5). The final section (8) covers Reporting recommendations. 
Table 1: Performance test matrix

[Tests are denoted Primary (P) or Secondary (S) for the two classes of sensors. Although all 11 tests are considered important for characterization of seismometer performance, the Primary tests are considered basic for Acceptance Testing purposes. $\mathrm{BB}=$ broadband, $\mathrm{WM}=$ weak motion, $\mathrm{SM}=$ strong motion]

\begin{tabular}{|c|c|c|c|}
\hline \multirow[b]{2}{*}{ Test } & \multirow[b]{2}{*}{ Description } & \multicolumn{2}{|c|}{ Importance, P or S } \\
\hline & & $\begin{array}{l}\text { WM BB } \\
\text { Velocity }\end{array}$ & $\begin{array}{c}\text { SM } \\
\text { Acceleration }\end{array}$ \\
\hline 1. Power demand & $\begin{array}{l}\text { Current drawn from DC power supply, start- } \\
\text { up transient and steady-state. }\end{array}$ & $\mathrm{P}$ & $\mathrm{P}$ \\
\hline 2. Sensitivity & $\begin{array}{l}\text { Basic scale factor, Volts per ground motion } \\
\text { unit. Also known as midband transfer func- } \\
\text { tion magnitude. }\end{array}$ & $\mathrm{P}$ & $\mathrm{P}$ \\
\hline $\begin{array}{l}\text { 3. Frequency response } \\
\text { and bandwidth }\end{array}$ & $\begin{array}{l}\text { Magnitude and phase versus frequency or } \\
\text { period. - } 3 \mathrm{~dB} \text { bandwidth based on frequen- } \\
\text { cy response. Commonly expressed as an S- } \\
\text { plane pole-zero model. }\end{array}$ & $\mathrm{P}$ & $\mathrm{P}$ \\
\hline 4. Self noise & Noise spectrum appropriately measured. & $\mathrm{P}$ & $\mathrm{P}$ \\
\hline $\begin{array}{l}\text { 5. Clip level and operat- } \\
\text { ing range }\end{array}$ & $\begin{array}{l}\text { Maximum measurable signal. Operating } \\
\text { range combines this hard clip with self noise } \\
\text { from Test } 4 \text { in a standard computation and } \\
\text { plot format. Test } 6 \text { measures soft clipping. }\end{array}$ & $\mathrm{P}$ & $\mathrm{P}$ \\
\hline 6. Linearity and distortion & $\begin{array}{l}\text { Output voltage versus input ground motion } \\
\text { as a function of the latter's amplitude. Qua- } \\
\text { litative waveform fidelity versus amplitude; } \\
\text { soft clipping is the amplitude at which li- } \\
\text { nearity departs specifications. }\end{array}$ & $\mathrm{S}$ & $\mathrm{S}$ \\
\hline $\begin{array}{l}\text { 7. Orientation and ortho- } \\
\text { gonality }\end{array}$ & $\begin{array}{l}\text { Orientation of reference axis of sensor. Or- } \\
\text { thogonality of sensor } \mathrm{X}, \mathrm{Y} \text {, and } \mathrm{Z} \text { outputs. } \\
\text { Misalignments between case reference } \\
\text { marks and true axes. }\end{array}$ & $\mathrm{P}$ & $\mathrm{P}$ \\
\hline $\begin{array}{l}\text { 8. Temperature effect on } \\
\text { sensitivity, offset, and } \\
\text { mass position }\end{array}$ & $\begin{array}{l}\text { Change in sensitivity and offset as a func- } \\
\text { tion of temperature. }\end{array}$ & $\mathrm{P}$ & $P$ \\
\hline $\begin{array}{l}\text { 9. Power supply voltage } \\
\text { effects }\end{array}$ & $\begin{array}{l}\text { Change in sensitivity and offset as a func- } \\
\text { tion of power supply voltage. }\end{array}$ & $\mathrm{s}$ & $\mathrm{s}$ \\
\hline $\begin{array}{l}\text { 10. Power supply noise } \\
\text { effects }\end{array}$ & $\begin{array}{l}\text { Change in noise as a function of power } \\
\text { supply noise (typically high-frequency } \\
\text { noise). }\end{array}$ & $\mathrm{s}$ & $\mathrm{s}$ \\
\hline $\begin{array}{l}\text { 11. Integration to dis- } \\
\text { placement of known dis- } \\
\text { placement or tilt inputs }\end{array}$ & $\begin{array}{l}\text { Double integrated acceleration for known } \\
\text { displacement input signal on a precision } \\
\text { shake table or step/tilt appliance. }\end{array}$ & $\mathrm{P}$ & $\mathrm{P}$ \\
\hline
\end{tabular}




\section{Definitions}

Standard definitions for inertial sensor terminology are found in IEEE Standard 528-2001 (IEEE Standard for Inertial Sensor Terminology). These are generally applicable to broadband seismometers and are used in these guidelines except as noted below.

Specific terms used in these guidelines for broadband seismometer and accelerometers are defined or explained below.

- Acceptance Test

Tests or inspections performed by a purchasing agency under a procurement or purchase contract for technical acceptance of sensors received from a vendor. Acceptance testing is generally done by the purchasing agency or its agent. Pre-award acceptance testing may be more rigorous than post-award acceptance testing.

- Broadband Seismometer (BB)

A sensor designed for earthquake ground-motion measurement over much of the wide range of frequencies of interest to seismology and earthquake engineering, usually from about 0.01 to $50 \mathrm{~Hz}$.

- $\quad$ Bandwidth

The part of the seismic frequency band where a particular sensor is intended to operate. Specifically, it is defined herein by the lower and upper frequencies at which the frequency response magnitude is $3 \mathrm{~dB}$ below the maximum value and within which the ripple is otherwise not larger than $\pm 1 \mathrm{~dB}$.

- $\quad$ Clip Level

The output level at which a sensor's output voltage deviates from the input waveform. There are two types of clipping, soft and hard:

o The soft clip level is the amplitude at which the sensor deviates by more than 1 percent from linear midband response.

o The hard clip level is the level above which the output cannot be pushed, or the level corresponding to the maximum voltage output of a seismometer or accelerometer.

There always will be a difference between soft and hard clip levels. While this difference is normally very small, even undetectable, in a few cases there will be a large difference. Also, it should be noted that, especially for weak-motion BB seismometers, it is possible for internal clipping to occur within the feedback loop. This internal clipping condition may not be obvious by observing only the output.

- Cross-Axis Sensitivity

Sensitivity of any axis to ground motion orthogonal to that axis. It is difficult to measure and to separate cross-axis sensitivity from misalignment error, so will not be further addressed in this document. 
- Distortion (Non-linear)

Deviation of the output signal from a sinusoidal waveform when the input is a pure sine wave.

- Exploratory Test

Tests or inspections done for purposes other than acceptance or qualification of instruments for procurement purposes. Exploratory testing may be done as part of research and development of sensors or to characterize the performance of an existing sensor.

- Frequency Response

The ratio of sensor output to motion input as a function of frequency for a particular sensor axis. Typically this response is measured as the complex transfer function between output and input and expressed as magnitude and phase spectra. For seismic sensors, it is commonly modeled in the S-plane as a transfer function, which is a complex Laplace transform and expressed using pole-zero representation.

- Linearity

A sensor property that describes the straight line goodness of fit between the measured sensor output and a sinusoidal input.

- Low Noise Model

A generally accepted model of the lowest seismic noise observed. One such model is the USGS New Low Noise Model (NLNM; Peterson, 1993). Another example is the GSN Low Noise Model (Berger and others, 2004).

- NIST

National Institute of Standards and Technology, a branch of the U.S. Federal Government that has responsibility for United States national standards of weight and measure.

- NIST-Traceability

A calibration or test is considered "NIST-Traceable" if and only if the primary test equipment used in the calibration has formal calibration paths leading back to primary United States standards of mass, length, temperature, time, or frequency.

- Operating Range

The portion of the ground motion spectrum between the clip level and the self noise of a seismometer, where the latter is represented as the root mean square (rms) of a sliding half-octave window. Operating range depends on the bandwidth in which the noise is measured.

- Orientation

The direction of motion that produces the maximum positive output from a particular sensor axis. The sensors covered in this document are three-component sensors in a right-handed Cartesian (XYZ) sense. Sensor channels are often presented in ZYX order (for example, Up, North, East). In use, orientation for a sensor is relative to the case, with external reference markings indicating axis directions. There will always be a small difference between the true and caseindicated orientations of sensors. 
- Orthogonality

The property that the three-component outputs of a sensor measure in mutually perpendicular directions or components of motion (XYZ in the right-handed Cartesian system).

\section{- Qualification Test}

Tests or inspections done to verify that a sensor meets specific criteria and is therefore qualified as a satisfactory product. Qualification tests are generally done by a vendor in response to agency-specific criteria or general market expectation.

\section{- $\quad$ Self Noise}

The electronic and mechanical noise at the output of a seismometer or accelerometer in the presence of zero input ground motion. Alternately, it can be the portion of a seismometer output signal not related to ground or case motion.

\section{- Sensitivity}

The ratio of output voltage to input motion (velocity or acceleration) for one sensor axis. For a sensor with a flat frequency response in middle of its pass band, sensitivity will be the value of this flat portion of the frequency response magnitude. For a non-flat frequency response, it is necessary to define sensitivity at a particular frequency. For this document, units of sensitivity for analog output sensors will be volts per meter per second $(\mathrm{V} / \mathrm{m} / \mathrm{s})$ for velocity and volts per meter per second per second $\left(\mathrm{V} / \mathrm{m} / \mathrm{s}^{2}\right)$ for acceleration, or for digital output sensors counts per meter per second (counts/m/s) for velocity and counts per meter per second per second (counts $/ \mathrm{m} / \mathrm{s}^{2}$ ) for acceleration.

\section{- Signal-to-Noise Ratio (SNR)}

The ratio of signal power to noise power in a particular frequency band, typically expressed in $\mathrm{dB}\left(10 \log _{10}[\{\right.$ signal power $\} /\{$ noise power $\left.\}]\right)$ defined over a specific frequency band.

- Strong Motion (SM)

Earthquake ground motions with amplitudes high enough to be felt by people and to affect the built environment. SM is typically measured using accelerometers. Typical peak amplitude range is $0.01-40 \mathrm{~m} / \mathrm{s}^{2}(0.001-4 \mathrm{~g})$ and associated frequency range is $0.1-50 \mathrm{~Hz}$.

- SM Accelerometer/Acceleration Sensor

A sensor with output proportional to acceleration within the frequency and amplitude ranges considered to be SM.

- Weak Motion (WM)

Earthquake ground motions with amplitudes below human perception. WM can be measured in terms of acceleration, velocity, or displacement, but velocity is most common. Nominal peak amplitude range is $10^{-10}-10^{-2} \mathrm{~m} / \mathrm{s}$ and nominal frequency range is $0.001-50 \mathrm{~Hz}$.

- Weak Motion Broadband Velocity Sensor

A sensor with output proportional to velocity within the frequency and amplitude ranges considered to be WM. 


\section{General Recommendations}

\subsection{Qualifications}

The personnel performing or reviewing broadband seismometer or accelerometer performance testing should be qualified by an appropriate combination of education, training, and experience to perform such testing. Specific experience with the use of comparable sensors should be a requirement. For acceptance and qualification testing, criteria for experience should be stated in any application-specific test procedures and personnel qualification should be documented in test reports.

The test facility should also be suitable for performing any specific test procedure. Facilities needed may vary for the different performance tests detailed in sections 6 and 7 . For example, an ordinary urban test laboratory may be sufficient for measuring many aspects of SM acceleration sensor performance, but the measurement of WM velocity sensor performance, especially self noise and operating range, may require a seismically quiet remote test laboratory. For acceptance and qualification testing, any special test facility qualifications should be stated in application-specific test procedures and should be documented in test reports.

\subsection{Quality Control}

Some level of quality control is advisable for all seismometer performance testing.

Exploratory testing under these guidelines may not require rigorous or formal quality control due to the research nature of such testing. However, as a minimum, good laboratory practice should be followed and testing should be documented adequately (well enough that another expert in the field can understand all salient points of the tests and procedures used).

Both qualification and acceptance testing should be done under a higher level of quality control. Vendors should have either formal quality assurance or quality control (QA/QC) certification (such as ISO 9001) or an internal system of procedures and documentation to control quality in sensor performance testing. Agencies or institutions involved in acceptance testing should also consider formal laboratory certification but at a minimum should have an internal system of quality control procedures and documentation applicable to sensor performance testing.

An important part of quality control is test equipment calibration; recommendations for this are given in the next section.

\subsection{Test Equipment Calibration}

The primary test equipment used in testing under these guidelines should be calibrated using an external reference or standard. Calibration frequency for test equipment should be annual unless otherwise justified or required. In the United States, formal NIST-traceability is the generally accepted standard of metrology practice; NIST or equivalent traceability is strongly recommended for acceptance and qualification testing.

For sensor testing, the earth's gravitational acceleration (g) can be considered a primary standard with a value of $9.80665 \mathrm{~m} / \mathrm{s}^{2}$ or, preferably, a precisely measured or accurately estimated 
(for example, http://www.ngs.noaa.gov/cgi-bin/grav_pdx.prl) local value, which may differ from that global standard value by several parts per thousand.

Any reference sensors used in performance testing should be at least as good in performance as the sensor being tested for the particular test. Reference sensors should be annually calibrated, with NIST-traceability where possible.

\subsection{Sample Size}

For acceptance and qualification testing, the testing of multiple samples of a sensor is highly recommended. The recommended minimum number of samples is three. Note that, in smallsample statistics, one typically needs a minimum of eight replicate samples to establish, with cross-validation, a 95 percent confidence interval for the median; three replicate samples may establish a 75 percent confidence interval for the median (Wilks, 1962); Rousseeuw and Verboven (2002) recommend using and demonstrate that the Median Absolute Deviation from the Median (MAD), corrected for sample size, may be used a substitute for the spread parameter (that is, standard deviation for a Gaussian distribution) for four or more replicate measurements.

\subsection{Physical Inspection}

Prior to performance testing, a sensor sample should always be inspected for its physical condition. This sample should include inspection of the following:

- Identification (model and serial number verification);

- External appearance;

- Visual inspection of enclosure, base, and connectors for defects, cracking, or corrosion;

- Gentle shake/roll to listen for internal mechanical problems; and

- Internal visual inspection (if appropriate or allowed) for defects, loose parts, or corrosion. Physical inspection should be documented in the test report, using photographs as appropriate.

\subsection{Test Conditions}

Sensor performance may be a function of environmental conditions such as temperature, pressure, and humidity. Except when environmental sensitivity is being specifically tested, environmental conditions during sensor testing should represent a nominal value, not an extreme. Environmental conditions should be noted and documented in the test report.

Testing of WM BB velocity sensors (especially self noise, linearity and distortion, cross-axis sensitivity, and temperature testing, as described in section 6) requires a special environment that has stable temperature and low ambient seismic noise. For example, the underground test facility at the USGS Albuquerque Seismological Laboratory (ASL) has temperature variations on the order of $0.05^{\circ} \mathrm{C}$ and noise levels only a few $\mathrm{dB}$ above the USGS New Low Noise Model (NLNM; Peterson, 2003). Low ambient seismic noise may also be needed for self noise testing of SM acceleration sensors. A preferable test site would be an underground seismic vault having stable temperature and minimal air movement. In-band seismic noise from all nearby sources should be minimized or eliminated. Electromagnetic noise sources should also be minimized or 
eliminated, if necessary, by shielding. In most cases it will not be possible to have ambient seismic noise below the self noise of the WM seismometer.

Typical office or laboratory conditions are generally acceptable for the SM accelerometer tests as described in section 7. In such an office or laboratory environment, care should be taken to minimize radio frequency interference (RFI) and electromagnetic interference (EMI) interference in the test area. Self noise testing of low-noise SM accelerometers typically requires a quiet site, with noise levels within a few $\mathrm{dB}$ of the NLNM, similar to that needed for the WM BB velocity sensors.

Physical conditions may also affect sensor performance during performance testing. Examples of potentially relevant physical conditions are floor/pier construction, sensor mounting, vibration, acoustic noise, sunlight (heating from), and air currents. These conditions should be carefully considered, controlled as necessary, and documented as appropriate.

Electrical interconnections should be compatible with manufacturer specifications for both the sensors and all test equipment. Proper grounding and power should be used. Grounds should use large cross-section copper conductors and single-point star topology to minimize ground loops and voltage noise from ground currents.

\subsection{Test Equipment}

The specifications of measurement and recording devices used in broadband seismometer testing should be sufficient to resolve, at appropriate levels, all parameters to be measured in determining whether sensors meet performance requirements and specifications. Lesser test devices can still be used to place worst-case bounds on performance criteria and to determine acceptance or qualification without determining full sensor performance.

Unless otherwise specified, digitizers having at least 21 bits of resolution at 200 samples per second (sps) and at least 22 bits of resolution at 20 or 40 sps should be used for all tests in which it is necessary to digitize and record analog waveforms.

Shake tables will be needed for some of the performance tests. For SM accelerometer testing, such tables should be of the precision linear slide or air-bearing types. Shake tables may be used to test the distortion of WM BB sensors as long as one accurately measures the position (or velocity or acceleration) of the table during the distortion test.

A step-calibration table (for example, Wielandt-designed, Lennartz Electronic in Germany see http://www.lennartz-electronic.de/MamboV4.5.2/index.php), along with appropriate analysis software, is useful for testing of WM BB velocity sensors. See appendix 1 for detailed application of a step-calibration table.

Especially for WM BB velocity sensors, a well-calibrated, stable, low-noise, reference WM $\mathrm{BB}$ sensor is useful for several of the tests. Reference sensors are also useful for SM instruments testing.

\subsection{Acceptance Testing Recommendations}

This category of testing will generally be done as part of procurement by an agency, independently of the vendor. Although these guidelines may be referenced within procurement documents, such procurement documents and agency requirements will take precedence over these 
guidelines. However, the following general recommendations for acceptance testing are provided as basic guidance:

- A high level of quality control and review is needed for measurements and reporting.

- Acceptance criteria should be provided for each of the tests performed, most appropriately as part of procurement documentation.

- If acceptance testing includes multiple samples, acceptance criteria should include statistical criteria to be applied to the aggregate result of each test (for example, mean, median, $\mathrm{min} / \mathrm{max}, 2$ sigma, worst case). The recommended minimum number of samples is three. Note that, in small-sample statistics, one typically needs a minimum of eight replicate samples to establish a 95 percent confidence interval for the median.

- If there are formal QA/QC procedures and systems in place, these should be followed in the testing and reporting.

- If there are no formal QA/QC procedures and systems in place, then testing should be reviewed internally by at least one qualified person.

- Procedural provisions should be made in advance regarding what actions are to be taken if sensor sample(s) fail to meet test criteria, either by mechanical/electrical failure or by design. There should be a rejection and retest plan within the test procedure that clearly states if and how corrections can be made and retesting done.

\subsection{Qualification Testing Recommendations}

This category of testing will generally be related to procurement but will be done by a vendor or a vendor's agent and the results reported to a procuring agency. It is recommended that application-specific qualification test procedure or procedures be written based on these guidelines and followed in the sensor testing.

The following general suggestions are provided as basic guidance:

- Application-specific qualification test procedures should be prepared by the vendor and approved in advance by the purchasing agency, if practical.

- Qualification testing demands a high level of quality control and review that is externally validated. Formal QA/QC procedures and systems are strongly recommended for the group performing the testing.

- Qualification criteria should be provided in the application-specific procedures for each of the tests performed, either by the purchasing agency or in accepted standards.

- If testing requires multiple samples, qualification criteria should include statistical criteria to be applied to the aggregate result of each test (for example, mean, min/max, 2 sigma, worst case).

- If there are formal QA/QC procedures and systems in place, these should be followed in the testing and reporting.

- If there are no formal QA/QC procedures and systems in place, then testing should be witnessed and test results and reports should be reviewed externally by at least one independent qualified person in addition to any internal review.

- The test procedure should contain provisions for repair and retesting in case of failures. 


\section{Recommended Testing for Weak Motion Broadband Velocity Sensors}

These test recommendations apply to broadband feedback seismometers (also referred to as "sensors" here) whose output is proportional to earth velocity and commonly used in WM earthquake monitoring networks. These sensors generally have velocity limits of approximately 0.001 to 0.02 meters per second.

A note on the use of calibration inputs: Many BB seismometers produce orthogonal X, Y, and $\mathrm{Z}$ outputs along the axes of a Cartesian coordinate system by internally summing the outputs of mechanical sensors whose sensitive axes are aligned along different axes (for example, $U, V$, and $\mathrm{W}$ axes, the "Galperin" arrangement). Calibration inputs for such sensors will typically excite equivalent ground motion along the $\mathrm{U}, \mathrm{V}$, and $\mathrm{W}$ axes, not the $\mathrm{X}, \mathrm{Y}$, and $\mathrm{Z}$ axes. For tests requiring the use of calibration inputs, the suggested procedure assumes that the test engineer will connect the test signal in a manner that will produce the desired output signal on each of the $\mathrm{X}, \mathrm{Y}$, and $\mathrm{Z}$ outputs. Also, the procedure assumes that the test engineer will apply any required "calibration enable” signal.

\subsection{Power Demand, Start-up and Steady-State}

\subsubsection{Purpose}

The purpose of this test is to measure initial and steady-state power supply current and voltage required by sensor. This test may also be used to quantify power consumption during warm-up/start-up time and to characterize the power consumption of any motors used during the sensor set-up phase (for example, locking, unlocking, and mass-centering motors).

\subsubsection{Test Equipment}

- Recording digital voltmeter (DVM), data logger, or equivalent, with capability to record at least 20 sps. The minimum resolution of the DVM or data logger shall be 4.5 digits or 16 bits.

- Ammeter capable of measuring up to 1 amp, with minimum resolution of 3.5 digits.

- Precision (1 percent) 1 ohm wire-wound resistor with 2 watt power dissipation capability

- Adjustable regulated DC power supply with voltage range of $10 \mathrm{VDC}$ to $24 \mathrm{VDC}$ and current capability of at least 1 amp.

\subsubsection{Setup and Procedure}

Insulation and air shielding is not necessary for this test. The sensor should be accurately leveled. With the power supply turned off, connect the sensor to a power supply capable of supplying the appropriate voltage and current. Also connect appropriate voltage and current monitoring equipment. A voltmeter and ammeter are appropriate for steady-state measurements. If transient start-up currents are to be measured, use a recording DVM or data logger operating at 20 sps. To measure current with the DVM or data logger, connect the 1- $\Omega$ resistor in series with the power supply input and use the DVM or data logger to measure the voltage across this resistor. 
If sensor has manual mass lock/unlock mechanism, unlock the masses at this point. Apply power. Record start-up current and voltage during power-up. If sensor has motor-driven lock/unlock mechanism, initiate the unlock sequence and record current and voltage until masses are fully unlocked. If sensor has motor-driven mass-centering mechanism, initiate the masscentering sequence and record current and voltage until masses are well centered. Keep sensor powered up for an additional 15 min under quiescent conditions (no lock/unlock or centering motors operating). Measure or record the steady-state current and voltage during the last minute of this 15-minute quiescent operating period.

\subsubsection{Analysis}

This analysis assumes that the power supply voltage remains stable within 2 percent for all current values. Determine the maximum current and time required to settle to steady-state from current measurements during the initial power-up. Determine the maximum and steady-state (if applicable) current for each mode of operation from current measurements during lock/unlock motor operation and mass-centering motor operation. Steady state current during motor operation only applies if the motor or motors in question operate continuously for more than $10 \mathrm{~s}$. Determine from current measurements during the last $1 \mathrm{~min}$ of quiescent operation the steady-state current requirement.

\subsubsection{Results}

Results should be presented in tabular form or graphical form, as appropriate. For example, transient start-up current $I$ could be presented in graphical form, as a function of time. If voltage fluctuates more than 2 percent, instantaneous voltage measurements taken simultaneously with current measurements should be used to calculate power demand. Power is calculated as voltage times current at each measurement time. Minimum results to be presented are:

- Maximum start-up current and power,

- Maximum lock/unlock motor start-up current and power,

- Quiescent lock/unlock motor current and power (if any motor runs more than $10 \mathrm{~s}$ ),

- Maximum mass-centering motor current and power,

- Quiescent mass-centering motor current and power (if any motor runs more than $10 \mathrm{~s}$ ), and

- Quiescent operational current and power (after 15 min of quiescent operation)

\subsection{Sensitivity and Offset}

\subsubsection{Purpose}

The purpose of this test is to determine midband sensitivity of sensor in $\mathrm{V} / \mathrm{m} / \mathrm{s}$. The midband frequency is defined as $0.17 \mathrm{~Hz}$ (approximately at the peak of the six-second microseism band). It is suggested that the tests described in section 6.10 be performed immediately after this test because the setup is nearly identical. Offset will also be determined.

\subsubsection{Test Equipment}

- Calibration table, Wielandt design or equivalent (Lennartz, 2006) or well-calibrated broadband reference seismometer. 
- Data logger with 20-bit resolution (broadband at 20 sps) or better, \pm 20 volt peak differential input, and capability to record a minimum of six channels at 20 sps or faster, with digital sensitivity (counts per volt) known to within 0.1 percent on all six channels.

- Reasonably quiet seismic pier with fairly stable temperature and little or no air motion.

- Reference sensor (method 2 only).

- Temperature measuring device with $0.1^{\circ} \mathrm{C}$ resolution and accuracy.

\subsubsection{Setup and Procedure}

Method 1: Calibration using a calibration step table. An example procedure for using this method to calibrate standard gain or high gain STS-2 seismometer is given in appendix 1.

Method 2: Calibrate by recording normal seismic background simultaneously with a wellcalibrated reference seismometer.

For method 2, set up the seismometer to be tested near the reference sensor in a quiet seismic vault which has a stable temperature environment. Both seismometers should be well insulated and shielded against air currents. Both should be on the same rigid table with the table attached to the earth at three points to ensure equal motion at both sensors. Connect both the reference seismometer and the test seismometer to the data logger. Record data for at least 12 hours in order to allow both seismometers to stabilize and produce good ambient-noise data.

For both method 1 and method 2, measure the temperature of the seismometer case. This information will be used later in section 6.10 .

\subsubsection{Analysis}

Method 1: Use the analysis procedure given in appendix 1. This requires DISPCAL (Wielandt, 2008) and TILTCAL (Wielandt, 2005) analysis software or equivalent.

Method 2: Using the known transfer function model (Laplace domain poles and zeros) developed in section 6.3 or supplied by the seismometer manufacturer, compute corrected power spectral densities (PSDs) for each channel of both instruments. These corrected PSDs should represent ground acceleration or velocity at the input to the sensor. For the seismometer under test, use the midband sensitivity supplied by the manufacturer as a trial value. Compare the PSDs across a common midband frequency band centered on the "six-second microseism" band. In most cases, the 0.17 to $0.34 \mathrm{~Hz}$ band will be appropriate. The PSD level of each test seismometer channel should be within one percent $(0.01 \mathrm{~dB})$ of the PSD level of the corresponding channel of the reference seismometer. If not, adjust the midband sensitivity value of the test seismometer until its corrected PSD level matches that of the reference seismometer. This adjusted midband sensitivity represents the measured sensitivity of the test seismometer.

For both methods: Determine the average DC offset value in volts for each channel of the test seismometer during quiescent conditions. This offset may be determined by calculating the mean value (in volts) of the seismometer output for 5 min of stabilized data.

\subsubsection{Results}

For both methods: Report the midband sensitivity value (at $0.17 \mathrm{~Hz}$, for example) for each channel in $\mathrm{V} / \mathrm{m} / \mathrm{s}$. Also report the DC offset value for each channel and the average seismometer case temperature for the same time segment at which this DC offset value was measured. 


\subsection{Frequency Response and Bandwidth}

\subsubsection{Purpose}

The purpose of this test is to determine the transfer function (amplitude and phase) for each component (X, Y, and Z) of the sensor.

\subsubsection{Test Equipment}

Method 1:

- Signal generator capable of producing sine waves from $0.001 \mathrm{~Hz}$ to $100 \mathrm{~Hz}$, logarithmically swept sine wave signal over the same frequency range, or random noise (for example, random white noise or pseudo-random binary sequence) spanning that band.

- Data logger with 20-bit resolution or better, \pm 20 volt peak differential input, and capability to record a minimum of four channels at 200 sps or faster, with digital sensitivity (counts per volt) known to within 0.1 percent on all channels.

Method 2:

- Digital-recording dynamic signal analyzer capable of frequency domain analysis from $0.001 \mathrm{~Hz}$ to $100 \mathrm{~Hz}$, which has a minimum of two channels, and a built-in signal source with the same capabilities described for the signal generator in method 1 (Agilent 35670A, Hewlett Packard 3562A, Stanford Research Systems SR785, or equivalent).

\subsubsection{Setup and Procedure}

This procedure assumes that the seismometer to be tested has a calibration coil or other calibration input that can be used to input a voltage or current that produces a known force on the proof mass or equivalent feedback-loop offset, either mimicking ground acceleration.

Method 1: Set up the seismometer to be tested in a quiet seismic vault having a stable temperature environment. Insulate and shield the seismometer from air currents and allow the sensor to settle to a stable temperature (generally takes hours to days). Connect signal source to calibration input of seismometer and, if a voltage, to channel four of data logger. Current-input calibrations will have to be buffered to the data logger by some means, such as measuring the voltage across a known resistance in the current path (for example, Section 6.1.2).

Connect the three $(\mathrm{X}, \mathrm{Y}, \mathrm{Z})$ outputs of seismometer to data logger. The amplitude of the calibration signal from the signal source should be set to a level that produces a good signal-tonoise ratio (SNR) (for example, 50 percent of full-scale, at the seismometer outputs) but does not produce clipping or obvious distortion. For velocity seismometers, this amplitude may depend on frequency because, typically, the calibration input of a seismometer produces an equivalent ground motion that is proportional to acceleration, not velocity.

It may be more practical or even necessary to test the upper and lower corners and midrange of a sensor separately since sampling rates, sample durations, and signal source capabilities can vary over the four or more decades of frequency measured by many modern seismic sensors.

Discrete frequency calibration_1a: If discrete-frequency sine waves are used, input sine waves at a sufficient number of frequencies so that the shapes of the band corners can be resolved. For example, if the seismometer is expected to have a $-3 \mathrm{~dB}$ point at $10 \mathrm{~Hz}$ on the high frequency end, one should calibrate at approximately 9, 10, and $11 \mathrm{~Hz}$ to properly identify the 
shape of this corner. In midband, the calibration frequencies need not be as close together. For example, if the seismometer has expected $-3 \mathrm{~dB}$ points at $10 \mathrm{~Hz}$ and $0.01 \mathrm{~Hz}$, the midband calibration frequencies can be approximately 5, 1, 0.5, 0.1, and $0.05 \mathrm{~Hz}$. Beyond the $-3 \mathrm{~dB}$ points, calibrate with a sufficient number of frequencies to identify the asymptotic response slopes. For seismometers with a high frequency corner greater than about $40 \mathrm{~Hz}$, it may be necessary to use a data logger with a sampling rate higher than 200 sps. Be sure to let each frequency run long enough that any transients between frequencies die out sufficiently to allow accurate analysis. Unless the calibration input is a voltage rather than a current and a series capacitor is used as described under "Swept sine calibration" (below), it will be necessary to adjust the source signal to smaller amplitudes at lower frequencies to avoid clipping or distortion.

Swept sine calibration_1b: If a calibration voltage input to the seismometer normally produces equivalent ground acceleration, it will be necessary to insert a large-valued capacitor in series with the calibrator input. The capacitor should be of the solid dielectric type. A suggested value is 22 microfarads. It is important that this capacitor, along with the ohmic input impedance of the calibrator input, produce a high-pass filter whose corner frequency is higher than the highest frequency to be applied. This is known as a "capacitive calibration" and will have the effect of producing equivalent ground velocity so that the amplitude of the swept sine signal will not need adjustment during the sweep. If capacitive calibration is used, ensure that the data logger channel being used to monitor the calibration signal is connected so that it directly monitors the output of the signal generator. Alternatively, to be sure of monitoring a voltage that is directly proportional to calibration current, one may monitor the voltage across a small series resistor (a few ohms) inserted into the calibration return line. If the upper and lower corner frequencies of the seismometer response are more than a factor of 100 different, it may be necessary to perform two different swept sine runs, each of which contains frequencies appropriate for measurements near each corner frequency.

Random noise calibration_1c: This type of calibration will be most useful in characterizing the low frequency corner of the response. However, if a data logger with sufficiently high sample rate is available, it may also be useful in characterizing the high frequency corner of the response in a separate test. In order to avoid clipping and distortion, it may be necessary to use a series capacitor so that calibration signals as seen by the seismometer are proportional to ground velocity (see "Swept sine calibration" above). The duration of this type of calibration should be at least four times the longest period tested and the sample rate 6 to 10 times the highest frequency tested.

Method 2: Set up the seismometer to be tested in a quiet seismic vault having a stable temperature environment. Insulate and shield the seismometer from air currents and allow the sensor to settle to a stable temperature (generally takes hours to days). Connect the signal source measured at the seismometer input to channel one input of the analyzer. Connect the output of one channel of the seismometer to channel two of the analyzer. Run a logarithmically swept sine wave calibration with the sweep frequency range set to cover frequencies from one-half the lower frequency corner to twice the upper frequency corner (or narrower, overlapping bands covering the same range). Assuming the use of a two-channel signal analyzer, it will be necessary to perform three separate runs-one for each seismometer channel. An example of this procedure is given in appendix 2 . 


\subsubsection{Analysis}

Method 1a: Analyze the recorded calibration input data (data logger channel four) and the recorded output from each seismometer channel (data logger channels one through three) through the use of CALEX (Wielandt, 2007) time domain analysis software. This program determines system parameters, such as corner periods, damping constants, and poles and zeros of the transfer function of analog filters or seismometers by modeling the response of the system to an arbitrary input signal in the time domain and fitting it to the observed output signal.

Methods 1b and 1c: Analyze the recorded calibration input data (data logger channel four) and the recorded output from each seismometer channel (data logger channels one through three) to determine output velocity amplitude and phase for all frequencies tested. In the case of the velocity swept sine and random noise calibration (using a series capacitor at the calibration input), dividing the Discrete Fourier transform (DFT) of the output by the DFT of the input will yield phase and amplitude information. The resulting phase and amplitude data should then be used to check (and fit, as necessary) the Laplace transfer function model $(H(s))$ provided by the manufacturer.

Method 2: Obtain and store the amplitude and phase response data from the signal analyzer for each seismometer channel. Some signal analyzers will allow the user to download amplitude and phase numbers for each frequency, or the creation of a hard copy plot of the amplitude and phase response plots. For some older analyzers (HP3562A, for example), it may be necessary to manually determine and write down the numbers from a screen plot. If the signal analyzer is able to fit a model transfer function, $H(s)$, to the measured response, this should be done. If not, it may be necessary to separately fit a model $H(s)$ to the measured data.

\subsubsection{Results}

For any of these methods, it is suggested that both measured data (amplitude and phase as a function of frequency) and fitted models $(H(s)$ ) be reported in the form of plots as well as numerically. Along with the midband sensitivity (and its frequency, serving as the transfer function normalization frequency) measured in section 6.2, $H(s)$ represents the transfer function of the seismometer. The poles and zeros of $H(s)$ should also be given in tabular form. Plots should be in velocity units $(H(s)$ poles and zeros should be given in rad/s and sensitivity plots for BB velocity sensors generally in $\mathrm{m} / \mathrm{s}$ ). Note that a seismic sensor has different transfer functions for displacement, velocity, and acceleration; these are trivially related to each other by a factor of omega.

\subsection{Self Noise}

\subsubsection{Purpose}

The purpose of this test is to determine the self noise of each channel of the seismometer. It is suggested that the test described in section 6.12 be performed immediately after this test because the setup is nearly identical.

\subsubsection{Test Equipment}

Method 1 (two-sensor test):

- Quiet seismic vault. 
- Reference WM BB sensor, well calibrated and known to have low self noise.

- Data logger with 23-bit resolution or better, \pm 20 volt peak differential input, and the capability to record at least six channels at 1 and either 40 or 200 sps, with digital sensitivity (counts per volt) known to within 0.1 percent on all channels. The highest sample rate used will determine the highest frequency that can be observed. It is strongly recommended that a single data logger be used so that all samples are taken simultaneously. Calculated self noise levels may be elevated if samples are not simultaneous (small phase errors between channels may damage the analysis).

Method 2 (three-sensor test):

- Quiet seismic vault.

- One reference WM BB sensors, well calibrated and with known low noise (and two test sensors).

- One or more data loggers having a total of nine channels with 23-bit resolution or better, \pm 20 volt peak differential input, and capability to record at 1 sps and either 40 or 200 sps, with digital sensitivity (counts per volt) known to within 0.1 percent on all channels.

For both methods, it is strongly recommended that environmental parameters such as wind speed, air pressure, and temperature (especially temperature within the seismometer test vault) be recorded or documented in some other way.

\subsubsection{Setup and Procedure}

Both methods below assume that the test seismometers are powered by DC power supplies set at the nominal power supply voltage recommended by the manufacturer. Sensor power may be provided by the data logger or a separate power supply. If sensor power comes from the data logger, make sure that it is a regulated voltage with low noise and ripple, because power-supply noise may increase sensor noise. For both methods described below, it is important that test personnel not be present in the seismic vault during the recording of background noise. All "cultural noise" and other external noise sources should be kept to a minimum.

Method 1: Two-sensor test. Set up reference seismometer and test seismometer in a quiet seismic vault near each other (preferably within $1 \mathrm{~m}$ ). The two seismometers should be carefully oriented according to their major axis alignment marks so that their (active) $\mathrm{X}$-axes are pointed the same direction within $0.1^{\circ}$, if possible. (The $\mathrm{X}$-axes of both seismometers may be pointed due East, for example, or may be aligned to any common reference line on the vault, pier, or slab surface.) Both seismometers should be placed on a dense granite slab (if available) that is supported on the vault floor or pier at three points only. One way to do this is through the use of three lead pads to support the slab. This method will produce better coherence between sensors and more accurate self noise values since even a good vault exhibits noise (especially tilt) incoherence over very short distances. Insulate and shield both sensors and the stone slab, if used, against air currents. Record data from all three channels of both sensors on the data logger for a period of time sufficient to allow the noise levels of both sensors to stabilize. This settling time may be several days in the case of BB sensors having a low frequency corner of $0.01 \mathrm{~Hz}$ or less. 
Method 2: Three-sensor test. Same as for two-sensor test, except that there will be two test seismometers instead of one. All three seismometers should be placed on a stone slab (if available) that is supported on the vault floor or pier at three points only. This method produces better coherence between sensors and more accurate (lower) self noise values.

\subsubsection{Analysis}

Method 1: Two-sensor test. Use the method of Holcomb (1989), or equivalent.

Method 2: Three-sensor test. Use the method of Sleeman and others (2006), or equivalent.

Comments: The three-sensor method typically produces lower self noise estimates than the two-sensor method, especially when SNRs are high (12 dB or higher), as one would expect in the 6-s microseism band. With both methods, noise estimates are better when longer time segments are analyzed (A.T. Ringler, U.S. Geological Survey, written commun., 2009). The three-sensor method is recommended over the two-sensor method unless only two sensors are available. Also, results are better when sensors having roughly the same noise level are compared.

\subsubsection{Results}

For both methods, provide in graphical form the derived power spectral density (PSD) noise levels, as a function of frequency, of all three channels of both the reference seismometer and the test seismometer or seismometers in terms of ground acceleration or velocity. Spectral analysis parameters and methodology should be documented, including ensemble size, overlap, and windowing. These noise levels should also be plotted as rms values on the ampORD operating range plots specified in Section 6.7.

\subsection{Self Noise Settling Time}

\subsubsection{Purpose}

The purpose of this test is to determine the amount of time required for the seismometer to settle to approximately its lowest noise state in the long period band. This test is intended to measure the decay of "popcorn" noise (also known as "glitches" or "pings") over time. Popcorn noise may be defined as sudden step changes in acceleration associated with relaxation of internal stresses. Thermally induced mechanical noise also closely mimics the " $1 / f$ noise" (noise that increases with the inverse of frequency) and primarily affects low frequencies. This test may be performed as part of the Self Noise Test in Section 6.4.

\subsubsection{Required Equipment}

The required equipment is the same as for the Self Noise Test in Section 6.4.

\subsubsection{Setup and Procedure}

The setup and procedure is the same as for the Self Noise Test in Section 6.4

\subsubsection{Analysis}

Perform power spectral density (PSD) analysis of long (at least 32,000 s) non-overlapping time segments of the 1 sps data beginning at the time of installation of the sensor or sensors un-

der test. Calculate PSDs using the same method as in the Self Noise Test in Section 6.4.4. The purpose of the analysis is to determine the evolution of the power level at $0.01 \mathrm{~Hz}$ over time. 


\subsubsection{Results}

Plot the power level at $0.01 \mathrm{~Hz}$ as a function of time for each sensor channel. Spectral analysis parameters and methodology should be documented, including ensemble size, overlap, and windowing (and to the extent possible the parameters and methodology should be similar to those used to compute the PSD for an amplitude operating range diagram)

\subsection{Pressure Test}

\subsubsection{Purpose}

The purpose of this test is to determine the sensitivity of the seismometer to changes in barometric pressure (which can affect BB seismometers through proof-mass buoyancy and instrument-case strains). This test may be performed as part of the Self Noise Test in Section 6.4.

\subsubsection{Required Equipment}

The required equipment is the same as for the Self Noise Test in Section 6.4. In addition, a digitally recording microbarograph is required. The latter may be accomplished through the connection of an analog microbarometer to one channel of one of the test data loggers.

\subsubsection{Setup and Procedure}

The setup and procedure is the same as for the Self Noise Test in Section 6.4 In addition, the microbarometer shall be installed in the seismometer test vault near the seismometers under test.

\subsubsection{Analysis}

Seismometer pressure effects will be evident only for frequencies lower than about $0.1 \mathrm{~Hz}$, so it is only necessary to analyze the 1 sps data. Compute the relative transfer function estimate between the pressure recording and the recording of each channel of the seismometer or seismometers under test. Best results will be obtained during time periods of maximum barometric pressure activity, such as storms.

\subsubsection{Results}

Plot the relative transfer function (as a function of frequency) between barometric pressure activity and each test seismometer channel.

\subsection{Clip Level and Operating Range}

\subsubsection{Purpose}

The purpose of this test is to determine the clip level or distortion level of the seismometer as a function of frequency. These, along with the self noise level determined in section 6.4, help to define the operating range of the seismometer.

\subsubsection{Test Equipment}

- Data logger, minimum resolution 4.5 digits or 16 bits.

- Power supply appropriate for seismometer. 
- $\quad$ Sine wave generator.

\subsubsection{Setup and Procedure}

Set up test seismometer on pier or on floor in work area. Level seismometer and unlock and center masses. For this test, it may not necessary to set up in a quiet seismic vault, although this may be done if appropriate. Insulate and shield the seismometer from air currents, allow to settle (hours to days). Connect signal source to calibration input of seismometer and, if a voltage, to channel 4 of data logger. Current-input calibrations will have to be buffered to the data logger by some means, such as measuring the voltage across a known resistance in the current path (for example, Section 6.1.2).

Connect the three $(\mathrm{X}, \mathrm{Y}, \mathrm{Z})$ outputs of seismometer to three channels of data logger. If possible, also connect mass position outputs to data logger. For several different discrete frequencies from one-half the low frequency corner to twice the upper frequency corner: At each frequency, beginning with low amplitudes, gradually increase the amplitude of the calibration signal from the signal source until it just begins to produce either hard clipping or obvious distortion (soft clipping) on each of the three (X, Y, Z) outputs of seismometer (these levels may differ somewhat on the positive and negative sides of the signal swing but should be similar if the sensor is properly centered and compensated for static gravity inputs).

\subsubsection{Analysis}

At each frequency, convert the positive and negative voltage clip or distortion levels to meters per second using the sensitivity values determined in 6.2 for each seismometer channel. Observe the behavior of the mass positions for evidence of saturation before the BB output saturates without producing conspicuous distortions (especially at low frequencies).

\subsubsection{Results}

Report in tabular form the hard-clip or distortion (soft-clip) levels and frequencies (both if available) for each channel in volts and in meters per second. Report any clipping observed on mass position channels that occurs without obvious clipping or distortion on the BB outputs. Also, using the results of Sections 6.4 and 6.7, generate a root-mean-square (rms) amplitude operating range diagram (ampORD) as described by Evans and others (2009).

\subsection{Linearity and Distortion}

\subsubsection{Purpose}

The purpose of this test is to determine linearity and distortion of each channel of the seismometer. However, this problem is a complex task and one must normally be satisfied with an incomplete answer. The main limitations are:

- The standard method, the two-tone test, measures only low-frequency intermodulation products, and is insensitive to odd-order harmonic distortion. In its conventional form it ignores the frequency-dependence of distortions.

- The stimulus is normally applied as an electric current to the calibration coil. While in a linear system such a current is equivalent to a seismic acceleration; with respect to non- 
linearity it is not. Any reasonably designed feedback system of the force-balance type will simply adjust the feedback current so that it compensates the stimulus. The mechanical system does not experience the same forces as from a seismic signal. An electrical stimulus essentially probes the electrical feedback circuit, not the mechanical receiver or the electromagnetic transducer, which also may exhibit nonlinearity.

- A nonlinear response to cross-axis signals (which is the most likely form of cross-axis sensitivity) cannot be detected with an electrical stimulus.

- Broadband sensors can be tested for nonlinearity on a shake table but the method requires certain precautions and is limited to the vertical component in practice. The method has not yet been worked out sufficiently for standardization.

\subsubsection{Test equipment}

A low-distortion, two-tone oscillator is convenient for distortion testing but is not required. Two ordinary sine wave generators whose output signals are summed over a passive high-pass or band-pass filter network provide a suitable stimulus for a two-tone test.

Distortions in the test signal are irrelevant when the method of linear modeling is applied as in Section 6.3.4, method 1b. No extra test equipment is required in this case. Frequency response and nonlinearity can be measured in a single experiment.

The mechanical receiver of a seismometer can only be tested on a shake table. Although the table need not have a better linearity than the sensor, its usefulness may be limited by rotational motion (tilt), friction, and stray magnetic fields. Most shake tables produce too much tilt for testing horizontal sensors.

On a step table, sensor nonlinearity is expected to produce a systematic discrepancy between up-going and down-going steps. Such a discrepancy can clearly be observed with passive (electro-magnetic) seismometers but the effect may be too small to measure the nonlinearity of a good feedback sensor.

\subsubsection{Procedure and Analysis}

Two methods to separate nonlinear distortions from the main signal are available:

- The classical two-tone test where two short-period, sinusoidal signals interfere and their low-frequency intermodulation product is extracted by Fourier analysis or low-pass filtering.

- The method of linear modeling where the stimulus is recorded and a synthetic output signal is subtracted from the observed one so that distortions show up in the residual.

Both methods can be used with an electrical stimulus or with a shake table.

\subsubsection{Two-Tone Test With Electrical Stimulus}

The two-tone test uses two interfering sine waves, conventionally at 1.00 and $1.02 \mathrm{~Hz}$, whose combined peak amplitude is half of the clip level of the sensor. The output signal is checked for the presence of a signal at $0.02 \mathrm{~Hz}$, using Fourier analysis or a suitable low-pass or band-pass filter, digital or analog. The ratio of the rms signal amplitudes near 0.02 and $1 \mathrm{~Hz}$ is the distortion ratio, usually expressed in decibels. It depends on the test frequencies, normally according to 
a power law. The test must be repeated with other frequency pairs such as 2.00 and $2.02 \mathrm{~Hz}$ or 5.00 and $5.02 \mathrm{~Hz}$ in order to establish this law. Distortions in feedback seismometers increase at short periods because the feedback current for a given output signal is higher and the loop gain is lower.

The distortion ratio also depends on the beat (difference) frequency. It is however expected to be constant in terms of acceleration because distortion normally results from a nonlinear force or its electric equivalent in the feedback circuit. The ratio is then inverse to the beat frequency at the output of a broadband seismometer. This should be checked for two beat frequencies within the passband of the sensor. The ratio can then be predicted for other beat frequencies.

\subsubsection{Linear Modeling With Electrical Stimulus}

For this method, it is necessary to record the stimulus in addition to the output signal. A special cable or adaptor may be required for the digitizer input. Moderate distortions in the test signal are irrelevant. A swept sine wave is the most instructive test signal; it may be produced by manually turning the dial of a sine wave generator. Capacitive coupling into the calibration coil gives a constant-amplitude output signal in the passband of a broadband sensor (as in Section 6.3.3, method $1 \mathrm{~b}$ ). The peak amplitude at the output should again be half of the clip level. Analyze the signals with CALEX or similar software, as in Section 6.3.4, method 1a. The impedance of the calibration input together with the series capacitor forms a first-order low-pass filter for the calibration current which must be included in the transfer function, at least approximately as a time delay. Nonlinear distortions, normally at twice the signal frequency, can be recognized in the residual between the real and the synthetic output signal. This test shows harmonic distortion of all orders and thus complements a two-tone test but can also replace it. For that purpose, use a two-tone signal or simply vary the amplitude of a single sine wave manually and observe the resulting low-frequency components of the residual.

\subsubsection{Two-Tone Test on a Shake Table}

Here we make use of the fact that a nonlinearity in the motion of a shake table generates a spurious displacement signal at the beat frequency. The amplitude of this displacement is independent of the beat frequency; it would also be observed with a purely sinusoidal stimulus as a permanent offset of the time-averaged table position. Thus the acceleration-equivalent of this signal is proportional to the square of its frequency and can be made as small as we like by decreasing the beat frequency. When the spurious displacement is 1 percent and we perform a twotone test with the classical frequencies, 1.00 and $1.02 \mathrm{~Hz}$, then the acceleration signal at $0.02 \mathrm{~Hz}$ is 108 decibels below the acceleration signal at $1 \mathrm{~Hz}$; even the velocity signal is $74 \mathrm{~dB}$ lower. If this attenuation is not enough, we can use lower beat frequencies. At some point an intermodulation signal will remain whose acceleration amplitude cannot be reduced. Most likely, it will be seen as soon as the intermodulation can be distinguished from marine microseisms. This frequency-independent acceleration signal can only originate in the seismometer.

\subsubsection{Linear Modeling on a Shake Table}

The situation is even more favorable if we can record the actual motion of the table with a good displacement transducer and use the linear-modeling method for analysis. In doing so we subtract the measured distortion of the table motion from the output signal. Measuring the motion with 0.1 percent nonlinearity would reduce the distortion ratio of the test equipment by 
another $20 \mathrm{~dB}$. It is thus quite possible to test the vertical component of broadband seismometers for nonlinearity on a shake table. In a Galperin-style triaxial system, the vertical component is sensitive to imperfections in any of the three oblique components, and it is unlikely that the horizontal components would differ much from the vertical.

The linearity of the displacement transducer may be good enough to evaluate other test signals, like swept sine waves, in order to observe high-frequency harmonic distortions in the seismometer output as well. There is also the option of operating a reference seismometer side by side with the test instrument on the shake table and convolving both output signals to the same overall response. Even if distortions observed in the difference trace cannot always be safely attributed to one of the seismometers, their absence would suggest a good linearity of both instruments, especially if they are of different design.

\subsubsection{Caveats}

The two-tone test has the limitation that it gives the distortion as a percentage of the output signal. What the result means in terms of ground motion depends on the frequency response of the system. A given level of distortion is expressed by different numbers in terms of ground acceleration and in terms of ground velocity. With stimuli at 1.00 and $1.02 \mathrm{~Hz}$, acceleration distortion is lower than velocity distortion by a factor of $50(34 \mathrm{~dB})$. One must also consider that this test is typically made at a signal amplitude of half of the clip level of the seismometer at that frequency, which means that the test is made at different levels of ground motion for different sensors and frequencies.

\subsubsection{Reporting a Two-Tone Test}

Report the following:

- a short description or sketch of the test setup;

- the distortion ratio for a typical combination of test frequencies (such as 1.00 and 1.02 $\mathrm{Hz}$ ) and beat frequency (the difference between test frequencies, or $0.02 \mathrm{~Hz}$ in this case);

- whether this ratio refers to the output signal, ground velocity, or ground acceleration;

- the peak amplitude of ground motion (velocity or acceleration) equivalent to the test signal;

- estimated power laws for the dependence of the distortion ratio on the test and beat frequencies, or measured distortion ratios at some other test frequencies (such as $2 \mathrm{~Hz}$ and $5 \mathrm{~Hz}$ ) and at one other beat frequency (such as $0.01 \mathrm{~Hz}$ ); and

- any other observation that might indicate a nonlinearity; document such observations with plots. 


\subsection{Orientation and Orthogonality}

\subsubsection{Purpose}

The purpose of this test is to determine the orientation of the true sensitive axes of the $\mathrm{X}, \mathrm{Y}$, and $\mathrm{Z}$ outputs of the seismometer. This information will also yield the orientation of the axes relative to each other, allowing the determination of their orthogonality.

\subsubsection{Test Equipment}

- Quiet seismic vault.

- Reference WM BB sensor for which the orientation of the sensitive axes is known within $0.5^{\circ}$ and mutually orthogonal within $0.5^{\circ}$.

- Data logger with 20-bit resolution or better, \pm 20 volt peak differential input, and the capability to record a minimum of six channels at 1 sample per second.

\subsubsection{Setup and Procedure}

This test may be performed as part of Section 6.2, so set up as in Section 6.2.3, method 2. Record data for at least 3 hours (preferably much longer) during quiet conditions (no earthquakes, no wind, no local cultural activity). Normally, recording overnight will meet these conditions.

\subsubsection{Analysis}

Using the LINCOMB3 software package (Wielandt, 2008), represent the output signals $\mathrm{X}_{0}$, $\mathrm{Y}_{0}, \mathrm{Z}_{0}$ of the reference seismometer (assumed to be exact) as a linear combination of those of the seismometer under test, $X_{1}, Y_{1}, Z_{1}$. That is, to determine a $3 \times 3$ matrix $\mathbf{S}$ with $\left(X_{0}, Y_{0}, Z_{0}\right)=\mathbf{S} \times$ $\left(\mathrm{X}_{1}, \mathrm{Y}_{1}, \mathrm{Z}_{1}\right)$. For this analysis, it is strongly recommended that the bandwidth be limited to the marine-microseismic band (approximately 0.1 to $0.25 \mathrm{~Hz}$ ). Higher and lower frequency noise may not be fully coherent between sensors.

\subsubsection{Results}

Report orientation errors between true active axes of the seismometer being tested and external reference markings. If it is deemed to be useful, also report the orientation of each axis of the test seismometer relative to the orientation of the reference seismometer.

\subsection{Temperature Effect on Sensitivity, Offset, and Mass Position}

\subsubsection{Purpose}

The purpose of this test is to determine the effect of temperature change on midband sensitivity and broad band data output offset, including effect on mass position.

\subsubsection{Test Equipment}

- Quiet seismic vault.

- Data logger with 20-bit resolution or better, \pm 20 volt peak differential input, and the capability to record a minimum of six channels at 1 sample per second and one auxiliary channel for recording temperature. 
- Reference WM BB seismometer.

- Temperature measuring device with minimum resolution of $0.1^{\circ} \mathrm{C}$.

- Low wattage heat source such as a 10 watt or 25 watt light bulb, DC powered.

- Cooling source such as a thermoelectric cooling device, capable of removing at least 10 watts of heat.

\subsubsection{Setup and Procedure}

This set-up is nearly identical to that of method 2 of Section 6.2, with the addition of a method for changing the temperature of the test seismometer. Set up the reference and test seismometers adjacent to each other in a quiet seismic vault and connect them to the data logger. Insulate and shield the reference seismometer. Do not insulate the test seismometer, but do shield it from air movement with a thin, thermally conductive air shield. This thermally conductive air shield will be used along with heating and cooling devices to slowly change the temperature of the seismometer without making it excessively noisy. The temperature measuring device should be taped to the surface of the test seismometer. If this device produces a voltage proportional to temperature, connect it to the auxiliary channel of the data logger. If the temperature measuring device has only a digital or analog readout, it will be necessary to write down the steady-state temperature for each step of this test.

Sensitivity has already been determined in Section 6.2 at ambient temperature. Turn on the heating device and allow the seismometer temperature to stabilize. This may require 24 hours or more. If temperature is not being recorded on the data logger, write down the temperature. Record at least 1 hour of data (including mass position voltages) from the reference and test seismometers. Turn off heating device, turn on cooling device and repeat. If possible, use two different power settings on both the heating and cooling devices so that sensitivity and offset may be determined at five different temperatures.

\subsubsection{Analysis}

For each temperature, analyze recorded data from each seismometer channel for sensitivity using method 2 of Section 6.2.4 (comparison of corrected PSDs of reference and test seismometer). Analyze recorded data for offset value in volts. Analyze mass position data for temperature versus mass position offset.

\subsubsection{Results}

Report in both tabular form and graphic form the value of midband sensitivity and offset as a function of temperature. Report mass position offset versus temperature.

\subsection{Power Supply Voltage Effect on Sensitivity}

\subsubsection{Purpose}

The purpose of this test is to determine the effect of changes in power supply voltage on midband sensitivity. Note that this test is secondary because it is not likely that broadband feedback seismometers will have any change in sensitivity as a result of power supply voltage. 


\subsubsection{Test Equipment}

- Step calibration table, Wielandt design or equivalent (Lennartz, 2006) or well-calibrated broadband reference seismometer.

- Data logger with 20-bit resolution or better, \pm 20 volt peak differential input, and capability to record a minimum of six channels at 20 sps or faster, with digital sensitivity (counts per volt) known to within 0.1 percent on all six channels.

- Quiet seismic vault.

- Reference sensor (method 2 only).

- Adjustable regulated power supply with current capability sufficient for test seismometer.

\subsubsection{Setup and Procedure}

The setup and procedure are nearly identical to that of Section 6.2. Assuming that sensitivity was determined in Section 6.2 at the nominal power supply voltage, the sensitivity test will be repeated only for the maximum and minimum power supply voltages recommended by the manufacturer. Either method 1 or method 2 of Section 6.2 may be used.

\subsubsection{Analysis}

Perform analysis by either method 1 or method 2 of Section 6.2.4, as appropriate, for each power supply voltage.

\subsubsection{Results}

Report the midband sensitivity for each power supply voltage setting (three different voltages including nominal, minimum, and maximum).

\subsection{Power Supply Noise and Voltage Effects on Seismometer Noise}

\subsubsection{Purpose}

The purpose of this test is to determine the effects of significant variations in power supply voltage and noise on seismometer noise.

The following applies to sensors needing only single-sided power. For those requiring twosided power, modify these tests to apply mirrored signals to each side of the supply.

\subsubsection{Test Equipment}

Identical to test equipment in Section 6.4.2, plus an adjustable regulated-voltage power supply capable of providing required current to sensor at all voltages and of adding a small white noise signal to that supply voltage (for example, by driving a DC-responsive high-bandwidth power amplifier from a signal generator with these capabilities). The addition of white noise to a power supply voltage may also be accomplished by installing a small resistor in series with one side of the power supply and periodically shorting across the resistor with a relay driven by a random telegraph signal.

\subsubsection{Setup and Procedure}

Voltage Effects: Identical to Section 6.4.3. Either method 1 or method 2 may be used. Assuming that the noise and offset in Sections 6.4 and 6.2 were determined with the power supply 
of the test seismometer set to the nominal value, repeat the procedure for power supply settings at the minimum and maximum values specified by the manufacturer. Do not change the power supply voltage for the reference seismometer.

Noise Effects: Add white noise (containing frequencies up to $10 \mathrm{kHz}$ ) to power supply voltage at an amplitude level approximately 1 percent of the power supply voltage. This should be done at the nominal (mid-range) voltage of the power supply voltage range specified by the manufacturer.

\subsubsection{Analysis}

Voltage Effects: For each power supply voltage setting, determine self noise of test seismometer or seismometers using either method 1 or method 2 of Section 6.4.4, as appropriate. Also determine offset in volts for each test seismometer channel under quiescent conditions.

Noise Effects: For the time period when white noise was added to the power supply voltage, determine self noise of test seismometer or seismometers using either method 1 or method 2 of Section 6.4.4, as appropriate.

\subsubsection{Results}

Voltage Effects: For each power supply voltage setting, provide in graphical form the derived power spectral density (PSD) noise levels, as a function of frequency, of all three channels of both the reference seismometer and the test seismometer or seismometers in terms of ground acceleration or velocity. These noise levels may also be plotted on the operating range plots specified in Section 6.7. Also, for each power supply setting, report the steady-state DC offset voltages for each test seismometer channel.

Noise Effects: For the time period when white noise was added to the power supply voltage, provide in graphical form the derived power spectral density (PSD) noise levels, as a function of frequency, of all three channels of both the reference seismometer and the test seismometer or seismometers in terms of ground acceleration or velocity.

\subsection{Other Potential Tests}

The WM broadband velocity sensor performance tests detailed in the preceding sections, and the metrics they provide, are arguably the most important tests and results to users of these sensors. Results of other performance tests may be important to some users, especially for exploratory testing. A partial list is provided below, with many more potential tests covered in IEEE Standard 1293-1998. Details of these tests are beyond the scope of these guidelines, but generic guidance can be found in IEEE Standards 337-1972 and 1293-1998.

Partial List of Other Performance Tests for WM Broadband Velocity Sensors

- Temperature Range, Operating/Non-Operating

- Temperature Effect on Frequency Response

- Temperature Effect on Noise and Operating Range

- Pressure Effect on Offset

- Pressure Effect on Noise and Operating Range

- Humidity Effect on Sensitivity/Frequency Response/Offset/Noise 
- RFI Susceptibility

- Magnetic Field Effects on Offset and Noise (for a technique to perform the latter, see http://www.rz.uni-karlsruhe.de/ bi77/download/paperware/Poster/PosterDGG08.pdf)

- Clip Recovery Time

- Cross-Axis Nonlinearity

- Effects of Power Supply Voltage on Clip Level

- Effects of Grounding Techniques on Noise 


\section{Recommended Testing for Strong Motion Acceleration Sensors}

These test recommendations apply to both feedback and open-loop accelerometers commonly used in strong-motion seismic and earthquake-engineering studies and generally having acceleration limits of a few to several tens of meters per second per second and approximately flat response from $0 \mathrm{~Hz}$ (“DC”) to a corner frequency at or above the upper band limit of applicable specifications, typically $40-80 \mathrm{~Hz}$.

In the following, note the opportunities for combining tests: Sections 7.1 (power usage), 7.5 (self noise), and 7.10 (power supply and noise) can be combined because they use the same setup on a quiet pier. Sections 7.3 (frequency response), 7.4 (clipping), and 7.11 (double integration) can be combined if using a shake table; or Sections 7.3 (frequency response), 7.4 (clipping) if using centrifuge with tumble mechanism. Sections 7.2 (static sensitivity) and 7.7 (orientation) can be combined if using the rectilinear box. Sections 7.6 (distortion) and 7.8 (cross axis) can be combined on a quiet shake table driven with a low-distortion sinusoid.

\subsection{Power Demand, Start-up, and Steady-State}

\subsubsection{Purpose}

Determine initial and steady-state power-supply current and power at a nominal midrange drive voltage. Quantify start-up (initial operation and settling) and stabilized quiescent current and power draws and the time to stabilize current draw to within 1 percent of that quiescent level.

The following applies to sensors needing only single-sided power. For those requiring twosided power, modify these tests to track power on both sides of the supply.

\subsubsection{Test Equipment}

- Recording DVM, data logger, or equivalent, with capability to record at least 20 sps. The minimum resolution of the DVM or data logger shall be 4.5 digits or 16 bits.

- Ammeter capable of measuring up to 1 ampere (A), with minimum resolution of 3.5 digits or 12 bits, ideally sampling at least 20 times per second-for example, a digital storage oscilloscope monitoring the voltage across a wire-wound series resistor of high wattage and low resistance (order $1 \Omega$ ), as in Section 6.1.2, or a recording DVM, such as an Agilent 34970A or equivalent, set to integrate over one or two power-line cycles and to take and store a measurement at least 20 times per second.

- Adjustable low-ripple, low-noise regulated DC power supply with voltage range of 10 VDC to 24 VDC and current capability of at least 1 A and able to maintain its output voltage to within 2 percent at a given voltage.

- A hard surface in a seismically quiet location on which to place and level the sensor

\subsubsection{Setup and Procedure}

Place the sensor on the hard surface and connect it to the power supply with its voltage set near the middle of the allowed range (commonly about 12.5 VDC). 
Use the recording ammeter to record current as a function of time for $1 \mathrm{~min}$ or the time required for the sensor to settle to quiescent current usage, whichever is longer.

\subsubsection{Analysis}

Find the peak current during the start-up transient and also convert this to power (supply voltage times peak current). Measure the current draw at or after the point where current draw fluctuates by no more than 1 percent about its quiescent value, commonly near the end of the measured current time series. Measure the duration of the start-up current pulse as follows: in the time series find the start time (the power-on time) and the time after which current fluctuates by no more than 1 percent of its steady-state level; the difference between these times (in s) is the duration of the start-up transient.

\subsubsection{Results}

Report the peak current during the start-up transient in milliamperes (mA), the duration of this transient as described in Section 7.1.4 (in seconds), and the steady-state quiescent current draw (in $\mathrm{mA}$ ). Also report these two current draws multiplied by the supply voltage, that is, the power draw in milliwatts $(\mathrm{mW})$.

\subsection{Static Sensitivity, Offset, and Linearity}

\subsubsection{Purpose}

Measure the $0-\mathrm{Hz}$ ("DC”) or low frequency sensitivity and offset of the sensor under nominal operating conditions. Ideally, also measure the deviation from linear of these quantities as a function of input acceleration. In both cases, measure both positive and negative accelerations up to values that are considerable fractions of the full-scale accelerations measurable by the sensor, for example between $\pm 1 \mathrm{~g}$, as on a tilt table or, better, \pm 75 percent of full scale, as on a centrifuge.

\subsubsection{Test Equipment}

- A precision tilt table with accurate, independent angle measure (for example, a well calibrated reference accelerometer such as a Honeywell QA2000-200); the rectilinear box used in Section 7.7; or a low-noise centrifuge equipped with sensor-flip mechanism.

- If using the rectilinear box, then also an accurately flat hard surface which has been leveled to within 1 milliradian (mrad) of horizontal. Note that tilt angle of $1 \mathrm{mrad}$ will produce the equivalent of $1 \mathrm{mg}$ of horizontal acceleration.

- If using a centrifuge, accurately orient the centrifuge axis vertically (within $1 \mathrm{mrad}$ ). Orient the flip mechanism holding the sensor so that the sensor case axis is oriented along the horizontal radius of the centrifuge in alternating positive and negative directions to the same accuracy. Note that there will be a static 1-g cross axis force on the sensor and that horizontality must be closely maintained for the plane of rotation and the orientation of the sensor axis to this plane. In order for the sensor axis orientations to remain accurate during the test, a tight, quiet main bearing and careful installation are required.

- If using a tilt table, orient the axis of the table within $1 \mathrm{mrad}$ of horizontal and the true active axis of the sensor within 1 mrad of perpendicular to the table axis (the case axis is 
precise enough for orienting to the surface of the tilt table), which may be accomplished by the rotary alignment appliance described in Section 7.8.2, cross-axis test equipment.

- An accurate (0.1 percent) sampling voltage recorder with minimum resolution of 5.5 digits or 20 bits.

- A reference accelerometer or calibrated means of measuring tilt table angle or centrifuge rotation rate. The value of $g_{\text {Local }}$ also must be known to good accuracy ( 0.1 percent) for either the tilt table or rectilinear box methods.

\subsubsection{Setup and Procedure}

If using the rectilinear box placed on the leveled flat surface with case axes alternately placed in the upward and downward direction, measure the output of the sensor with the case axis so oriented.

If using the tilt table, set it to five or more angles producing an evenly spaced set of input accelerations (not angles) between positive and negative $1 \mathrm{~g}$, measuring the output of the sensor at each position. The set of input accelerations should be symmetric about zero within a few gals $\left(\mathrm{cm} / \mathrm{s}^{2}\right)$.

If using a centrifuge, run the centrifuge at five or more speeds set to yield accelerations evenly spaced from 0 -g to about 75 percent of maximum sensor range. At each speed measure the output of the sensor with the axis pointed outward, and again with the sensor pointed inward (or tumbling through a $360^{\circ}$ range with vertical axis). The center of mass of sensor pendulum should remain at a constant radius from centrifuge rotational axis (to within 0.1 percent) when changing orientation.

For both tilt table and centrifuge, co-mount the reference accelerometer to provide a reliable measure of input acceleration.

For both tilt table and rectangular box, correct from local to global $\left(9.80665 \mathrm{~m} / \mathrm{s}^{2}\right)$ gravitational acceleration to within one part per thousand (using, for example, the United States gravity model at http://www.ngs.noaa.gov/cgi-bin/grav_pdx.prl, accessed 27 July 2009).

\subsubsection{Analysis}

Record sensor output at zero and at one or more pairs of approximately equal positive and negative accelerations.

- For the box test, only 0 and \pm 1 gLocal will be measured.

- In the centrifuge and tilt tests, measure at least five input accelerations including the maximum, zero (table stationary), and minimum input levels and values with half the accelerations of the two extrema. Ideally, measure two or more additional pairs of evenly spaced acceleration values. (Evenly spaced tilt angles do not provide evenly spaced acceleration inputs_-please use evenly spaced accelerations to support the subsequent fitting of a line to those data.) In all cases, measure at a set of input accelerations symmetrically disposed about zero using the reference accelerometer or accurate (independent measure of tilt angle or rotation rate) as the trusted measure of input accelerations. 
As needed, compute the true input accelerations for each measured sensor output, including corrections for local gravitational acceleration, rate and radius of centrifuge, and the reference accelerometer.

For the tilt table and centrifuge, the sensitivity is the slope of a best-fitting line in a plot of sensor output against true input acceleration. The offset will be the fitted sensor output model where this line crosses zero input acceleration. We recommend an L1 (minimization of the sum of absolute errors) fitting procedure rather than L2 (minimization of the sum of squared errors).

For the box test, the sensitivity is half the difference between the sensor output at corrected \pm 1 -g inputs (positive minus negative) and the offset is the mean of these two sensor output values.

Linearity can be derived from the tilt-table or centrifuge tests and is the largest observed deviation from the linear best fit as a percentage of the sensors full-scale input acceleration (\%FS).

\subsubsection{Results}

Report the sensitivity (in $\mathrm{V} / \mathrm{m} / \mathrm{s}^{2}$ ) and offset (in Volts) and if available the largest deviation of measured sensor output from the best fit linear estimate in terms of percent of full scale. Report the largest input accelerations used in terms of percent of full scale.

\subsection{Frequency Response and Bandwidth}

\subsubsection{Purpose}

Use a shake table (best at high frequencies) or centrifuge tumble test (best at low frequencies) to determine the amplitude and phase responses of the sensor, and to create an accurate Laplace model of same (poles and zeros in units of rad/s). From the Laplace model $(H(s))$ abstract the corners and bandwidth of the sensor - the flat portion of $|H(s)|(-3 \mathrm{~dB}$ corners; allowed ripple $\pm 1 \mathrm{~dB}$ ). In the case of low-damping (for example, shock) accelerometers, the upper corner is about 20 percent of the sensor's natural frequency but may be stated in terms of a frequency below which there is less than 1-dB positive amplitude error and moderate phase shift.

\subsubsection{Test Equipment}

- Shake table, preferably moving on a vertical axis to minimize tilt-induced errors in the result; should be able to input frequencies from one octave below to one octave above the flat band of the sensor. Ideally, should move on precision air bearings for minimum noise but a precision linear slide is generally adequate.

- Alternately, a centrifuge equipped with an accurately machined horizontal-plane tumbling mechanism that can cycle at varying frequencies (creating sine waveforms) across the sensor's flat band and beyond by at least one octave on both sides regardless of input acceleration.

- A combination of shake table and tumbling centrifuge may be optimal, taking advantage of the high-frequency fidelity of the former and low-frequency fidelity of the latter.

- A precise reference accelerometer co-mounted with the sensor under test (Honeywell QA2000-200 or equivalent) and with flat response over more than the required flat band of the sensor under test. 
- A recorder (minimum 20-bit resolution) recording the outputs of test and reference sensors continuously at 200 sps or more and with sample skew no more than 100 microseconds between channels.

\subsubsection{Setup and Procedure}

Rigidly co-mount the sensor being tested and the reference sensor to the stage of the shake table and case orient the active axes of both parallel to the active axis of the table, or similarly co-mount the test and reference sensors in the tumble fixture of the centrifuge at equal radii to the proof mass of each sensor (they will be stacked vertically with their proof masses along the axis of the tumble mechanism).

Input acceleration signals should have peak accelerations equaling about 25-75 percent of sensor full scale. Input-signal bandwidth should exceed that of the required flat-response band of the sensor under test by at least one octave beyond each end of the flat-response band.

For the shake table, either a swept sine wave or broadband noise may be used. For the centrifuge, a swept or stepped sine (a series of constant-frequency sine waves spaced at quarter or half octaves) may be used. Record identical intervals from each sensor for sine input ideally representing the same number of cycles (greater than 16) at each frequency.

\subsubsection{Analysis}

For noise or swept sine waves, compute discrete Fourier transforms (DFTs) of the output from the sensor under test and from the reference sensor.

Correct the latter for its transfer function if that function deviates significantly from flat amplitude and zero phase within the band of interest. In any case, scale the output of both sensors to SI acceleration units $\left(\mathrm{m} / \mathrm{s}^{2}\right)$.

Assuming that the reference sensor's transfer function is known, compute the complex ratio of test to reference DFTs and present this ratio as amplitude (in $\mathrm{V} / \mathrm{m} / \mathrm{s}^{2}$ ) and phase (in radians).

If constant frequency sine waves were used, compute amplitude and phase relative to the (corrected) reference sensor, respectively, for example, in the frequency domain by DFT peakamplitude comparisons and in the time domain by cross correlation. Collate these into amplitude and phase spectra.

In both cases, compute a set of Laplace poles and zeros (which define $H(s)$ ) which produce amplitudes and phases closely matching the observed spectra, preferably by an objective L1 bestfit method.

\subsubsection{Results}

- A log-log plot of measured amplitude response and $|H(s)|$ (in units of $\mathrm{V} / \mathrm{m} / \mathrm{s}^{2}$ against $\mathrm{Hz}$ )

- A semi-log plot of phase (in linear radians) against frequency (in log $\mathrm{Hz}$ )

- If manufacturer provides another model of $H(s)$, include this on both plots as well

- A listing of the poles and zeros (in rad/s) for best-fit and manufacturer's Laplace models

- If fixed-frequency sine waves were used, provide a listing of amplitude and phase at each input frequency 


\subsection{Clip Level}

\subsubsection{Purpose}

Using a shake table or centrifuge, dynamically determine the sensor's positive and negative soft- and hard-clip levels at one or more frequencies within the flat-response band of the sensor, for example, at about 0,1 , or $30 \mathrm{~Hz}$. Generally, the centrifuge may only be capable of generating lower frequencies and the shake table the higher frequencies, so a mix of techniques may be appropriate. Clipping levels may be a function of frequency, though it is constant across the pass bands of most accelerometers in seismic and earthquake-engineering use.

Soft clipping is the amplitude at which the sensor deviates by more than 1 percent from its linear static response. Hard clipping is the level above which the output cannot be pushed. In all cases, there will be at least a slight difference between these levels, though it may be too small to measure. In a few cases, there will be a large difference.

\subsubsection{Test Equipment}

- Shake table (horizontal or vertical) capable of generating accelerations greater than the specified hard-clip level at some in-band frequency (for example, $30 \mathrm{~Hz}$ ), or a low-noise centrifuge equipped with sensor-flip or -tumble mechanism (for example, "DC" or a few $\mathrm{Hz})$.

- If using a centrifuge (IEEE Std. 836-2001), accurately orient the centrifuge axis within $1 \mathrm{mrad}$ of vertical and the centrifuge table accurately orthogonal to this axis; accurately orient the flip- or tumble-mechanism (for example, secondary turntable) to orient the sensor axis along the radius of the centrifuge in alternating positive and negative directions; this mechanism should move in a horizontal plane (note that there will be a static 1-g cross-axis force on the sensor and that horizontality must be very closely maintained for the plane of rotation and the orientation of the sensor axis to this plane). Orientation of the pendulum pivot axis should normally be vertical but may be altered to suit the sensor; positioning the pendulum proof mass over the center of rotation of any flip/tumble mechanism is essential for measuring linearity at finite frequencies. Positioning this pendulum mass at an accurately known radius from the centrifuge axis is important to the accuracy of sensitivity measurements.

- Reliable co-mounted reference accelerometer oriented parallel to the test sensor axes and having hard-clip and distortion levels (soft clip) higher than the sensor under test (Honeywell QA2000-200 or equivalent).

- Either an integrating DVM or a continuous recorder (minimum resolution 5.5 digits or 20 bits); the former is appropriate only for quasi-static testing in a centrifuge.

\subsubsection{Setup and Procedure}

For the shake table, rigidly co-mount the sensor being tested and the reference sensor to the table with their axes parallel to the shake table motion axis. Drive the table with a sine wave at one or more in-band frequencies at which the table can exceed the specified hard-clip level of the sensor. First, drive the table with the peak acceleration somewhat below the specified clip level, ideally just below the soft-clip level (the level at which sensor linearity deviates from linear by more than about 1 percent of full scale). Second, drive to a peak acceleration about 10 percent higher than the clip level (taking care not to exceed relevant safety specifications). Additional 
input acceleration levels between soft and hard clip will provide a measure of distortion in this range.

With the centrifuge, drive at these same two or more levels, preferably with a tumble mechanism generating a sinusoidal output at some in-band frequency, for example, $1 \mathrm{~Hz}$. If using only a flip mechanism, alternate axis orientations to generate positive and negative static accelerations.

For the shake table or tumbling centrifuge, use the continuous recorder (having less than 100 microseconds sample skew) to record both the reference and test sensors to record at least several cycles of the sine wave. For the centrifuge with flip mechanism, use the DVM to record static acceleration outputs of each sensor in each orientation.

\subsubsection{Analysis}

Correct for sensitivity errors of both sensors and the continuous recorder.

If desired, the unclipped test provides an opportunity for a cross-correlation test between the test and reference sensors, which in turn provides a dynamic measure of linearity to complement the results in Section 7.2.

For the hard-clipped input, measure the output of the sensor under test during its interval of hard clipping. Plot a detail of the portions surrounding the start and end of hard clipping and including the level of soft clipping, putting both the test and reference sensors on the same graph at the same scale. This plot allows one to judge the level and manner in which the test output deviates from true input near the clipping level (the reference sensor will not be clipping); this soft clip will always be lower than the hard clip and create an output signal that is at least slightly rounded as it approaches and departs hard clip. Clip-recovery time may be evaluated in this manner as well.

\subsubsection{Results}

If the correlation test is performed, report the correlation coefficient for the unclipped large trace either here or in Section 7.2.

Report the test sensor output (in both $\mathrm{V}$ and apparent $\mathrm{m} / \mathrm{s}^{2}$ ) during hard clipping in each of the positive and negative directions. If a sine wave was input, present plots of both positive and negative clipping at the start and end of clipping regions of the waveforms, with the reference sensor waveform co-plotted, closely underlying, and at the same scale as the test sensor. That is, display the details of the waveforms from below soft clipping into hard clipping so that the test and reference sensor waveforms are easily compared. If static inputs are used, one should plot test sensor output against true acceleration input (from the reference sensor) across several points ranging from below soft clip to above hard clip. The latter can be combined with the sensitivity and linearity tests of Section 7.2 if using a centrifuge.

\subsection{Self Noise and Operating Range}

\subsubsection{Purpose}

Record a continuous sample of sensor output at a site and during an interval when monitored ambient seismic noise is well below the sensor's expected self noise. If a low-noise site is not 
available, or if the expected sensor self noise is below the ambient seismic noise, then either of the self noise measurement methods described in Section 6.4 may be used.

Using the method of Evans and others (2009), analyze this noise sample and the hard-clip level from Section 7.4 to determine amplitude operating range and rms noise spectral density (“ampORD” and “rmsSD”).

Note that the resulting operating range will overstate the low-distortion range of the sensor if soft clipping is significantly lower than hard clipping.

\subsubsection{Test Equipment}

- A hard surface at a very quiet site known to have ambient seismic noise below the selfnoise specification of the sensor under test. This surface will generally be a concrete pier on bedrock or the equivalent.

- Isolators are unlikely to be appropriate because they generally tilt at long periods.

- Piers in populated regions, along roads, near rotating machinery, in high RFI or EMI environments, or on soft sediments are unlikely to be quiet enough for this test.

- A more sensitive (generally weak-motion velocity) monitoring sensor similarly mounted at or near this test location.

- High-resolution continuous recorder or recorders operating at 200 sps and recording both the test and monitoring sensor outputs and having self noise well below the test sensor specification (minimum resolution 20 bits) and sample skew less than 100 microseconds.

\subsubsection{Setup and Procedure}

Mount (if appropriate also anchor) the test and monitoring sensors to the hard surface or surfaces.

Record their output simultaneously at 200 sps for a period of at least $5 \mathrm{~h}$, preferably from about 00:00 to 05:00 local civil time.

Select an interval of the test sensor record having a duration of at least $2^{18}$ samples (about 22 min) during which time the monitoring sensor shows neither vehicular or other significant cultural noise nor natural seismic events.

In some cases, particularly during winter recording, microseism noise will be measurable by the test sensor and will push up the apparent self-noise of that device at intermediate periods. In such circumstances, it is particularly important that the monitoring sensor be of high quality and that its noise spectrum be shown with all test sensor spectra to allow evaluation of valid and invalid measurement frequencies for the test sensor self noise.

\subsubsection{Analysis}

Process the record from the test sensor (and of the monitoring sensor if appropriate) with the MatLab software of J.R. Evans, C.R. Hutt, J.M. Steim, R.L. Nigbor, and E. Wielandt (written communication, 2009) or the equivalent, taking care to use identical windowing, weighting, and sample rates. Other inputs to this software are the hard-clip level of the test sensor and the relevant operating-range specifications (as frequency-decibel pairs covering the specified spectrum). 


\subsubsection{Results}

The two plots named ampORD and rmsSD and a list of measured (worst case) operating ranges and specified operating ranges for each frequency band in the specifications.

\subsection{Distortion}

\subsubsection{Purpose}

Using a very quiet shake table (preferably with air bearings or cross-flexure supports) driven by a low-distortion oscillator near $1 \mathrm{~Hz}$, place an upper limit on sensor on-axis total harmonic distortion (THD). It is unlikely that the sensor's true THD can be measured by this technique.

\subsubsection{Test Equipment}

- Very quiet shake table, preferably with air bearings or cross-flexure supports and that moves vertically.

- Low-distortion oscillator with sinusoidal output near $1 \mathrm{~Hz}$ or the fundamental mode of the shake table, but in either case well within the sensor's specified flat band. A more common signal generator in series with a narrow band-pass filter may be a viable alternative to a low-distortion oscillator.

- Reference accelerometer co-mounted with test sensor (Honeywell QA2000-200 or equivalent).

- Continuous recorder at 200 sps or more recording both test and reference sensors (minimum 20-bit resolution, sample skew less than or equal to100 microseconds).

\subsubsection{Setup and Procedure}

Case-align test and reference sensors in parallel with active axis of shake table. Begin driving shake table with oscillator and allow at least $1 \mathrm{~m}$ for table motion to settle out from all startup transients.

Record both sensors at 200 sps or more continuously for at least $2^{18}$ samples (about $22 \mathrm{~min}$ ).

\subsubsection{Analysis}

Compute the normalized PSD spectra with the same windowing and weighting used for self noise in section 7.5. The MatLab script GST_NormalizedWelch may be used for this purpose.

Compute the total power in the fundamental frequency (typically including a few adjacent bins to either side of the fundamental) and, separately, the total power in the next largest spectral harmonic frequency.

The THD limit is the ratio in decibels of the off-band power divided by the power in the fundamental.

The THD of the shake table can be similarly estimated from the reference sensor.

\subsubsection{Results}

The THD limit (in $\mathrm{dB}$ ); true THD is likely to be lower. Ideally, also present the PSD spectrum in log-log form $\left(\mathrm{m} / \mathrm{s}^{2}\right.$ versus $\left.\mathrm{Hz}\right)$ and the shake table THD estimated from the reference sensor. Note the harmonic used in the numerator, for example, $2^{\text {nd }}, 3^{\text {rd }}$. 


\subsection{Orientation (Case to Actual) and Orthogonality}

\subsubsection{Purpose}

Using an accurately machined rectilinear box fully containing the sensor and oriented to the case-indicated active axes (that is, "as used"), determine the orientation of each true active axis relative to the sensor case external reference marks (four-sided rolling flip about each axis when it is accurately horizontal). This test also yields sensor offset and sensitivity over a range of $\pm 1 \mathrm{~g}$ (see Section 7.2) but does not measure linearity. This test is performed on a precision-ground flat surface which has been leveled to within $1 \mathrm{mrad}\left(3.4 \operatorname{arc} \min\right.$ or $\left.0.06^{\circ}\right)$ of horizontal as measured with a precision machinist's level or equivalent.

\subsubsection{Test Equipment}

- Accurately leveled precision flat ground surface rigidly mounted to a bedrock pier or equivalent.

- Integrating 4.5-digit DVM, preferably recording to a computer in response to a manual trigger.

- Accurately machined rectilinear box (generally open on the face corresponding to the top of the sensor) with test sensor mounted to, contained within, and accurately case oriented to this box.

\subsubsection{Setup and Procedure}

Select and verify level for a portion of the flat surface at least as large as the rectilinear box. Place the box at this same location throughout the test.

Sequentially place the box on each of its six sides, record the output of each sensor while in each position (generally three orthogonal axes) and then reoccupy and record the output at the first of those orientations (to evaluate how much the sensor drifted during the test).

\subsubsection{Analysis}

The sensitivity and offset are found per Section 7.2.

Orientation of each true active axis relative to the corresponding case axis is found from the four values obtained when the respective case axis was horizontal.

Two opposite-side pairs are formed from these four measurements. The difference of each pair, scaled to g's by the previously determined sensitivity, equal the sine of the deviation angle between the case axis and the true active axis in that plane. Two such deviations and their vector sum (root of sum of squares) are the alignment errors of the axes and are direction sensitive.

The worst deviation from $90^{\circ}$ between axes, as computed from respective in-plane deviations, may be used as the orthogonality error. Additionally, one may also express these results with the three-by-three sensitivity matrix S relating observed output the input acceleration:

$\vec{a}_{\text {True }}=\mathbf{S} \vec{a}_{\text {Obs }}$. Matrix $\mathbf{S}$ is nearly diagonal; the off-diagonal terms express cross-axis terms due to axis misalignments. The (nearly always smaller) "parasitic” cross-axis terms inherent to a precisely oriented sensor are discussed in Section 7.8; these distinct contributions to effective crossaxis sensitivity are to be expressed separately. 
The difference between the first and seventh orientation, which should be identical, similarly translated to g's, $\mathrm{m} / \mathrm{s}^{2}$, or $\% \mathrm{FS}$ is the drift of the sensor during the test and should be at least one order of magnitude smaller than the signal differences in the orientation test.

\subsubsection{Results}

Report two orientation errors for each axis and the vector sum of this pair of deviations (in degrees and \%g). Report three axis-pair angular separations (in degrees) and their deviations from $90^{\circ}$. Report the drift or drift rate observed over the time span of the test.

\subsection{Translational Cross-Axis Sensitivity}

\subsubsection{Purpose}

Place an upper limit on sensor (parasitic) translation-to-translation cross-axis sensitivity (that is, the portion of cross axis sensitivity not due to simple axis misalignment).

This test generally should be performed simultaneously with the THD test of Section 7.6.

In Section 7.12, the cross-axis sensitivity of translational accelerometers to rotations should, ideally, also be quantified. Both rotational accelerations (that is, torque effects about the pendulum's pivot axis) and tilts (that is, long-period rotations in the horizontal plane subjecting the accelerometer to varying gravitational inputs) should be considered. In total there can be five cross-axis sensitivities for each sensor axis; however, such tests are presently beyond the scope of this Guideline.)

\subsubsection{Test Equipment}

- A very quiet shake table (preferably with air bearings or cross-flexure supports) moving vertically or horizontally when driven by a low-distortion oscillator or equivalent near 1 $\mathrm{Hz}$ (or the first fundamental of the table if it is within the flat band of the sensor).

- An adjustable turntable between the shaker and the sensor with which small angular alignment changes can be made (generally by way of a worm gear at the outer radius of the turntable).

- A high-resolution data logger operating at 200 sps or more and recording continuously (minimum 20-bit resolution and less than 100 microseconds sample skew).

- Digital storage oscilloscope.

\subsubsection{Setup and Procedure}

Mount the turntable rigidly to the shake table and the sensor rigidly to the turntable with the axis under test oriented perpendicular to the shake table axis.

Using the turntable while the shake table is in motion and monitoring the test sensor output on an oscilloscope, adjust the turntable slightly to minimize the visible response to the input oscillations. This step orients the true active axis perpendicular to table motion.

Allow the table to settle for at least $1 \mathrm{~min}$ and then record at least $2^{18}$ samples (about $22 \mathrm{~min}$ ) of data, as in Section 7.6. 


\subsubsection{Analysis}

Compute the PSD of this data segment as in Section 7.6. Take the ratio of the total power in the fundamental frequency ( $1^{\text {st }}$ harmonic) of the cross-axis test sensor (plus the power of the $2^{\text {nd }}$ harmonic, if appropriate, because it can be significant on a cross-flexure table since these often move as a parallelogram and axis alignment does not fully account for the secondary off-axis direction) to the power in the fundamental measured simultaneously by the on-axis component of the test or reference accelerometer (all scaled to $\mathrm{m} / \mathrm{s}^{2}$ ).

\subsubsection{Results}

Report this off-axis to on-axis power ratio (in $\mathrm{dB}$ ).

\subsection{Temperature Effect on Sensitivity and Offset}

\subsubsection{Purpose}

In an environmental chamber set successively to at least three (preferably five or more) widely spaced temperatures spanning the operational temperature range of the sensor, use a $\pm 1 \mathrm{~g}$ flip test to determine the sensitivity and offset of the sensor at each temperature. Fit linear or cubic polynomials to these values, which will yield models of sensitivity against temperature and offset against temperature.

\subsubsection{Test Equipment}

- Environmental test chamber large enough to contain the flip jig, the sensor under test, a counterbalance to the sensor (or identical second sensor), and able to generate and maintain temperatures across the operational range of the sensor.

- Flip jig which can accurately position one or more axes of the sensor in vertical up and vertical down positions; this jig should be supported on both sides of the sensors (preferably by bearings outside the chamber on both sides) and actuated manually or mechanically at user command.

- A 4.5-digit DVM (ideally a manually triggered recording variety to reduce operator transcription error).

\subsubsection{Setup and Procedure}

Place one or more sensors on the flip jig so that one or more case axes of each sensor are perpendicular to the jig axis. (In a three-component sensor, two axes can be tested in one run, with the third parallel to the jig axis.)

Attach a chamber-control temperature sensor to one of the sensor cases (or chassis) using heat-sink compound and mechanical or adhesive restraint so that it remains attached during the $360^{\circ}$ excursions of the flip jig. For each sensor, supply power from a source outside the chamber. For each axis under test, extract the output signals to a DVM outside the chamber. Take care to keep all cables free of encumbrance over the flip jig's range of rotations.

For each temperature (starting at the maximum and proceeding to the minimum to minimize condensation) allow the chamber to reach stable temperature as measured by the case sensor. Allow the sensor to soak at this temperature for some time so that its temperature is also stable. (The sensor case may be opened if not contraindicated to allow more rapid equilibration. Also, a 
well designed chamber allowed to overshoot and return to the set point generally can bring a small sensor to stability in under an hour. Large or massive sensors or integrated sensor-recorder systems (DASs) may take more time to equilibrate. Observe the internal sensor temperature if available, otherwise observe the sensor-case temperature sensor, and wait until this temperature remains constant within $\pm 1^{\circ} \mathrm{C}$ over a period of several minutes.)

Within a period of several minutes or less, position each case axis under test in positive and negative 1-g positions, allow the sensors to settle for a few moments, and then record sensor outputs with the DVM (0-g input accelerations are not used; those orientations only allow the other axis under test to be read at $\pm 1 \mathrm{~g}$ ).

(Care may be needed to prevent condensation on a cold sensor when chamber temperature is raised. Judicious use of desiccants and performing above-ambient tests before below-ambient can mitigate this tendency.)

\subsubsection{Analysis}

From each +1 -g and -1 -g output value, compute the sensitivity $\left(\mathrm{V} / \mathrm{m} / \mathrm{s}^{2}\right)$ from the difference of the two values and the offset $(\mathrm{V})$ plot as a function of temperature $\left({ }^{\circ} \mathrm{C}\right)$. Fit linear or, preferably, cubic polynomials to these values as a function of temperature.

\subsubsection{Results}

For each axis under test, provide a table listing temperature, sensitivity, and offset, and the polynomial coefficients of the best fit. For each axis, provide a plot of sensitivity against temperature and a plot of offset against temperature.

\subsection{Power Supply Voltage and Voltage-Noise Effects on Offset and Sensitivity}

\subsubsection{Purpose}

This test is designed to determine the effects on sensor offset and static sensitivity of significant variations in power-supply voltage and to determine the effect on sensor self noise of the presence of white noise in that supply. Test at least at a low, midrange, and high input voltage (for example, 11, 12.5, and 14 VDC), and preferably also at the sensor's specified limiting operational supply voltages. For the midrange supply voltage, test the effect of adding white noise having an rms equal to 1 percent of that supply voltage. Determine offset and sensitivity as a function of input voltage and the presence of noise, and compare sensor self noise with and without the white noise in the supply voltage.

The following applies to sensors needing only single-sided power. For those requiring twosided power, modify these tests to apply mirrored signals to each side of the supply.

\subsubsection{Test Equipment}

- Adjustable regulated-voltage power supply capable of providing required current to sensor at all voltages and of adding a small white noise signal to that supply voltage (for example, by driving a DC-responsive high-bandwidth power amplifier from a signal generator with these capabilities).

- An integrating 5.5-digit DVM (ideally a manually triggered recording type). 
- The rectilinear box and slab or comparable means of creating large, static accelerations symmetrically disposed about zero, for example, $\pm 1 \mathrm{~g}$.

- A high-resolution data logger operating at 200 sps or more and recording continuously (minimum 20-bit resolution and less than 100 microseconds sample skew).

\subsubsection{Setup and Procedure}

Assuming the box and slab method, rigidly attach sensor inside box and case aligned to it.

Set the power supply to the low voltage and start the sensor. Proceed as for Section 7.2.3 to measure sensor outputs in positive and negative acceleration positions. Change to a new supply voltage and repeat procedure until offset and sensitivity have been measured at three to five input voltages spanning the allowed operational range of the sensor.

Place the instrument in its normal operating orientation on a quiet pier. Set the supply voltage to the midrange level and add white noise to that signal (rms equal to 1 percent of the supply voltage). Proceed as in Section 7.5 to measure the sensor self noise for comparison to that determined in Section 7.5 with a quiet power supply.

\subsubsection{Analysis}

Compute sensitivity and offset at each supply voltage as in Section 7.2.4. Best fit linear or cubic polynomials as in Section 7.9.4, excepting that the horizontal axis is supply voltage rather than temperature.

Take the ratio (or $\mathrm{dB}$ difference) of the self-noise PSD spectrum determined here to that determined in Section 7.5.

\subsubsection{Results}

As in Section 7.9.5, provide a table of supply voltage, sensitivity, and offset as well as the coefficients of the fit polynomial. Provide a spectrum of the self-noise ratio in $\mathrm{dB}$ versus frequency.

\subsection{Double Integration of Band-Limited Displacement Square Wave}

\subsubsection{Purpose}

With the sensor in fully operational mode with proper power and case axis aligned parallel to the shake table, input band limited or otherwise rounded steps in displacement and determine whether the acceleration sensor output can be doubly integrated to reproduce the amplitude and waveform of this input displacement signal.

This test is highly effective for discovering whether numerous key parameters (linearity, sensitivity, distortion) are acceptably close to specified levels. Further, double integration is a task routinely performed with acceleration records and the sensor must be able to do this reliably. However, this test typically does not identify which particular parameter may be at fault if the test is failed.

Thus, the double integration test is a key primary test, which if passed lends broad confidence in the performance of the sensor and if failed generally requires further research to discover the cause. 


\subsubsection{Test Equipment}

- Shake table programmed to create a band-limited step in displacement at moderately high acceleration.

- Reference sensor or sensors for acceleration or table displacement, the latter preferable.

- A high-resolution data logger operating at 200 sps or more and recording continuously (minimum 20-bit resolution and less than $100 \mu$ s sample skew) and recording both reference and test sensor outputs.

\subsubsection{Setup and Procedure}

Co-mount test and reference sensors on axis with shake table.

Excite table with large-amplitude displacement square wave for which velocity, acceleration, and jerk, or bandwidth, have been limited to be within the sensors' capabilities. Square wave should have a period of about $10 \mathrm{~s}$ (that is, dwell time at each displacement extreme about $5 \mathrm{~s}$ ).

Record for at least $1 \mathrm{~min}$ and extract from this record a portion beginning about $20 \mathrm{~s}$ before the table comes to rest at zero displacement, through at least five cycles (preferably more), and ending about $20 \mathrm{~s}$ before the table again reaches the zero-displacement position.

\subsubsection{Analysis}

Correct the acceleration as needed for its transfer function.

Doubly integrate this signal to displacement and plot this displacement against the displacement measured by a reference displacement sensor or similarly computed from a reference acceleration sensor.

If a baseline correction is needed, use any common method which preserves the DC response of the accelerometer (DC generally must be preserved to generate matching waveforms in such broadband signals). The $20 \mathrm{~s}$ pre-excitation and post-excitation windows are intended to assist with accurately determining baseline corrections.

Displacement waveforms between reference and test sensors should match closely in shape and amplitude.

\subsubsection{Results}

Provide the plot of reference and test sensor displacement waveforms identically scaled and overlaid in the same frame of the plot. Ideally, also show test-sensor acceleration and velocity waveforms, any reference acceleration trace (same frame as corrected acceleration from test sensor) and, if a baseline correction was applied, that correction as it appears in acceleration.

\subsection{Other Potential Tests}

The 11 strong motion accelerometer performance tests detailed in the preceding sections, and the metrics they provide, are arguably the most important to users of these sensors and the resulting seismic and engineering data. Results of other performance tests may be important to some users, especially for exploratory testing. A partial list is provided below, with many more potential tests covered in IEEE Std. 1293-1998. Details of these tests are beyond the scope of these guidelines, but generic guidance can be found in IEEE Stds. 337-1972 and 1293-1998. 


\section{Partial List of Other Performance Tests for Strong Motion Accelerometers}

- Temperature Effect on Frequency Response

- Temperature Effect on Noise and Operating Range

- Humidity Effect on Sensitivity, Frequency Response, Offset, and Self Noise

- RFI and EMI Susceptibility

- Clip Recovery Time

- Rotational-to-translational Cross-Axis Sensitivity

- Baseline hysteresis (defined in IEEE Std. 528-2001) may be approximately tested by performing repeated excursions between \pm 1 g or levels of similar amplitude, returning the sensor to a precisely controlled horizontal $(0-\mathrm{g})$ position after each excursion. This test requires further development to eliminate subjectivity and variations in procedure, because the horizontal position must be reoccupied each time very precisely and the excursions should be well controlled and repeatable. 


\section{Reporting}

\subsection{Recommended Test Report Contents}

We recommend that test reports, especially those for the more formal acceptance and qualification testing purposes, contain the sections and order shown in table 2.

Section A should provide an overall test plan, simply stating what testing will be done to what sensors.

Section B should be a brief description of the test facility and a list of who is performing the tests, with their qualifications.

Section C should provide a list of test equipment, with manufacturer or manufacturers, model or models, serial number or numbers, facility identification numbers/codes (as appropriate), last calibration date or dates, and other identifying information.

Section D should provide calibration data or test reports; this information may be in an appendix.

Section E should contain a description of the sensor samples to be tested, including manufacturer, model, serial number or numbers, options, and other identifying information. Photographs are useful here. In addition, this section should contain results of the pre-test physical inspection described in Section 5.5.

Section F should describe the test conditions for all of the tests covered in the report. As a minimum, temperature and relative humidity should be stated. Other environmental and physical conditions relevant to evaluation of the results should be included.

Sections G-K should follow the test procedures given in Sections 6 and 7. If multiple sensor samples were tested, results for all sensor samples should be included in Sections I-K and should be summarized statistically and discussed in a Section $\mathrm{K}$ for each test type. These sections should be repeated for each distinct performance test.

Section L should provide combined results when multiple samples were tested.

Section $\mathrm{M}$ should contain a summary of all results from all tests of all samples. We recommend a tabular format.

Section N should describe any issues or problems encountered during the tests that affect the results. For example, repairs and retests should be documented here. Also included should be any deviations from the test procedures and any exceptions to the procedures or criteria (most pertinent for acceptance testing).

Section O should contain an overall evaluation of the results with respect to criteria. This section is appropriate for acceptance tests and perhaps for qualification tests, but may not be needed for exploratory tests.

Section P should describe the formal or informal quality control performed on the testing and the report. This information may include a description of the review process. It may include signatures of reviewers for more formal acceptance or qualification test reports. The following section expands on this recommended report section. 
Table 2: Recommended test report contents and organization

\begin{tabular}{|c|c|c|}
\hline Section & Title & Comments \\
\hline A & Overall Test Plan Summary & \\
\hline $\mathrm{B}$ & Test Facility and Personnel Description & \\
\hline $\mathrm{C}$ & Test Equipment & \\
\hline $\mathrm{D}$ & Test Equipment Calibration Data & May be in an appendix. \\
\hline $\mathrm{E}$ & Sensor Sample Description or Descriptions & \\
\hline $\mathrm{F}$ & Test Conditions & \\
\hline G & Test Setup & \\
\hline $\mathrm{H}$ & Test Procedure & \\
\hline I & Measurements & $\begin{array}{l}\text { Repeat } G-K \text { for each test } \\
\text { and } \mathrm{I}-\mathrm{K} \text { for each sensor. }\end{array}$ \\
\hline $\mathrm{J}$ & Analysis & \\
\hline K & Results & \\
\hline $\mathrm{L}$ & Combined Results & Each test, for multiple samples \\
\hline M & Summary of Results (all samples, all tests) & All samples, all tests \\
\hline $\mathrm{N}$ & Deviations and Exceptions (if needed) & If needed \\
\hline $\mathrm{O}$ & Evaluation (optional) & Optional \\
\hline $\mathrm{P}$ & Quality Control & \\
\hline
\end{tabular}




\subsection{Report Quality Control and Review}

\subsubsection{Acceptance Test Reporting}

This category of testing will generally be done as part of a procurement action by an agency, independent from the vendor providing the sensors. Therefore, a high level of quality control and review is needed for measurements and reporting.

It is beyond the scope of this guidelines document to recommend specific quality control or review procedures for specific procurements. Procurement documents and agency requirements will take precedence over these guidelines. However, the following general suggestions are provided as basic guidance:

- Acceptance criteria should be provided for each of the tests performed, most appropriately as part of procurement documentation. These criteria should be stated in the test report.

- If acceptance testing includes multiple samples, acceptance criteria should include statistical criteria to be applied to the aggregate result of each test (for example, mean, $\mathrm{min} / \mathrm{max}, 2$ sigma, worst case). These criteria should be stated in the test report.

- If there are formal QA/QC procedures and systems in place, these should be followed in the testing and reporting.

- If there are no formal QA/QC procedures and systems in place, then test reports and test data should be reviewed internally by at least one qualified person.

- Repair and retesting, if allowed and done, should be documented in the test report.

- Test data, raw and processed, should be archived as appropriate along with the report for future reference as-needed.

\subsubsection{Qualification Test Reporting}

This category of testing will generally be related to a procurement, but will be done by a vendor or a vendor's agent and the results reported to a procuring agency. Therefore, the results and reporting need a high level of quality control and review that is externally validated.

It is beyond the scope of these Guidelines to recommend specific quality control or review procedures for specific procurements. High-level quality programs such as ISO 9001 are adopted by many manufacturers to address such requirements. However, the following general suggestions are provided as basic guidance:

- Acceptance criteria should be provided for each of the tests performed, either by the purchasing agency or in accepted standards. These criteria should be stated in the test report.

- If acceptance testing requires multiple samples, acceptance criteria should include statistical criteria to be applied to the aggregate result of each test (for example, mean, $\mathrm{min} / \mathrm{max}, 2$ sigma, worst case). These criteria should be stated in the test report.

- If there are formal QA/QC procedures and systems in place, these should be followed in the testing and reporting.

- If there are no formal QA/QC procedures and systems in place, then test reports and test data should be reviewed externally by at least one independent qualified person in addition to any internal review. 
- Repair and retesting, if done, should be documented in the test report.

- As appropriate, test data, raw and processed, should be archived along with the report for as-needed future reference.

\subsubsection{Exploratory Test Reporting}

This category of performance testing covers less formal scientific and research and development purposes. It therefore does not demand the same level of formal quality control as do the other two categories. It is likely that the formality implied in Section 8.1 is not needed.

Nevertheless, it is recommended that testing be documented with some completeness and that a report should be generated and archived. It is further recommended that such a final test report, along with test data as appropriate, be reviewed internally by at least one qualified person prior to archiving. It may be that testing in this category will eventually be peer-reviewed as part of a publication process, but such peer review may not be a good substitute for internal review at the test report level (for example, because reviewer expertise in the subject might not be not verified).

\section{References}

These guidelines are the result of two workshops on the subject of seismometer testing, one in 1989 and the second in 2005. Documentation of these workshops is in the following two proceedings:

Hutt, C., Nigbor, R., and Evans, J., eds., 2009, Proceedings of the guidelines for seismometer testing workshop, Albuquerque, New Mexico, 9-10 May 2005 (“GST2”): U.S. Geological Survey Open-File Report 2009-1055, 48 p. (Also available at http://pubs.usgs.gov/of/2009/1055/.)

Hutt, C., 1990, Standards for seismometer testing-A progress report: Albuquerque, N. Mex., unpublished report available from Albuquerque Seismological Laboratory and included as an appendix to Hutt and others (2009).

There are several international standards published by IEEE (the Institute for Electrical and Electronic Engineers, www.ieee.org) describing the testing of inertial sensors. While these are intended for a broader scientific and engineering audience, portions are directly applicable to these Guidelines and provide consensus-approved terminology, methods, procedures, and specifications (the consensus of the engineers who wrote the IEEE standards). These standards are:

Institute for Electrical and Electronic Engineers, 1972, IEEE standard specification format guide and test procedure for linear, single-axis, pendulous, analog torque balance accelerometers: Los Alamitos, Cali., IEEE Standard 337-1972, 71 p.

Institute for Electrical and Electronic Engineers, 1998, IEEE standard specification format guide and test procedure for linear, single-axis, nongyroscopic accelerometers: Los Alamitos, Cali., IEEE Standard 1293-1998, 247 p. 
Institute for Electrical and Electronic Engineers, 2001a, IEEE standard for inertial sensor terminology: Los Alamitos, Cali., IEEE Standard 528-2001, 25 p.

Institute for Electrical and Electronic Engineers, 2001b, IEEE recommended practice for precision centrifuge testing of linear accelerometers: Los Alamitos, Cali., IEEE Standard 836-2001, 97 p.

Other References:

Advanced National Seismic System Technical Integration Committee, 2002, Technical guidelines for the implementation of the Advanced National Seismic System-Version 1.0, U.S. Geological Survey Open-File Report 02-0092, 92 p.

Working Group on Instrumentation, Siting, Installation, and Site Metadata of the Advanced National Seismic System Technical Integration Committee, 2008, Instrumentation guide lines for the advanced national seismic system: U.S. Geological Survey Open-File Report, 2008-1262, 41 p.

Berger, J., Davis, P., and Ekström, G., 2004, Ambient earth noise-A survey of the Global Seismographic Network: Journal of Geophysical Research, vol. 109, B11307 10.1029/2004JB003408, not paginated.

Evans, J.R., Followill, F., Hutt, C.R., Kromer, R.P., Nigbor, R.L., Ringler, A.T., Steim, J.M., and Wielandt, E., Method for calculating self-noise spectra and operating ranges for seismographic inertial sensors and recorders, 2010. (unpublished data, approved by USGS director for publication, in review at Seismological Research Letters).

Holcomb, L.G., 1989, A direct method for calculating instrument noise levels in side-by-side seismometer evaluations: U.S. Geological Survey Open-File Report 89-214, 35 p., accessed July 21, 2008, at http://earthquake.usgs.gov/regional/asl/pubs/files/ofr89-214.pdf .

Holcomb, L.G., 2002, Experiments in seismometer azimuth determination by comparing the sensor signal outputs with the signal output of an oriented sensor: U.S. Geological Survey Open-File Report 02-183, 205 p., accessed July 22, 2008, at http://pubs.usgs.gov/of/2002/ofr-02-0183/ofr-02-0183.pdf.

Lennartz Electronic, 2006, The CT-EW1 calibration table, document no. 990-0062: Tübingen, Germany, Lennartz Electronic GmbH, accessed December 18, 2009, at http://www.lennartz-

electronic.de/MamboV4.5.2/index.php?option=com_content\&task=view\&id=26\&Itemid=27.

Peterson, J., 1993, Observations and modeling of seismic background noise: U.S. Geological Survey Open-File Report 93-322, 94 p.

Rousseeuw, Peter J., and Verboven, Sabine, 2002, Robust estimation in very small samples: Computational Statistics and Data Analysis, v. 40, no. 4, p. 741-758.

Sleeman, R., van Wettum, A., and Trampert, J., 2006, Three-channel correlation analysis-A new technique to measure instrumental noise of digitizers and seismic sensors: Bulletin of the Seismological Society in America, v. 96, no. 1, doi:10.1785/0120050032, p. 258271. 
Wielandt, E., 2007, CALEX software package entitled, version 2007: Stuttgart, Germany, Institute for Geophysics, accessed October 30, 2009 at ftp://aslftp.cr.usgs.gov/pub/software/Wielandt_Software, and accessed October 30, 2009 at www.geophys.uni-stuttgart.de/downloads.

Wielandt, E., 2008, DISPCAL software package, version March 2008: Stuttgart, Germany, Institute for Geophysics, accessed October 30, 2009, at:

ftp://aslftp.cr.usgs.gov/pub/software/Wielandt_Software, and accessed October 30, 2009 at www.geophys.uni-stuttgart.de/downloads.

Wielandt, E., 2005, TILTCAL software package, version 2005: Stuttgart, Germany, Institute for Geophysics, accessed October 30, 2009 at ftp://aslftp.cr.usgs.gov/pub/software/Wielandt_Software, and accessed October 30, 2009 at www.geophys.uni-stuttgart.de/downloads.

Wielandt, E., 2008, LINCOMB3 software package, version 2008: Stuttgart, Germany, Institute for Geophysics, accessed October 30, 2009, at:

ftp://aslftp.cr.usgs.gov/pub/software/Wielandt_Software, and accessed October 30, 2009, at www.geophys.uni-stuttgart.de/downloads.

Wilks, S.S., 1962, Mathematical statistics: John Wiley \& Sons, 644 p. 


\section{Appendix 1: Step Table Calibration Procedure}

Note: This procedure is presented only as an example. It contains some instructions that are applicable only for use at the USGS Albuquerque Seismological Laboratory (USGS/ASL), such as data paths to USGS/ASL network drives.

\section{Wielandt Step Table Calibration for the STS-2 High or Standard Gain Seismometer}

The calibration will require:

- Wielandt step table with actuator box (modified to accept square wave input from function generator), bearing arm and tilt table,

- 12 volt direct current (VDC) power supply,

- digitizer for data recording,

- communications cables for data transmission,

- function generator,

- sensor to be tested, with host box,

- flathead screw driver,

- hex driver,

- DC volt meter, and

- foam box with cover.

\section{Contents:}

\section{Set-up}

A: Connections, cam selection, table level

B: Sensor placement, leveling, and electronic centering

i. Vertical component

ii. Horizontal components

\section{Function configuration and running of sequence}
A: Configuring DB360 function generator
$\mathrm{B}$ : Measuring $\Delta \mathrm{P}$
C: Running sequence

\section{Set-up}

\section{Part A: Connections, cam selection, table level}

Connect voltage standard calibrated digitizer (Q330) to power and communications.

Verify the Q330 is accessible with Willard.

Plug in function generator to AC power.

Plug in 9 VDC power supply to AC power. 
On step table: Proper cam must be selected for the instrument to be calibrated. Cams are labeled $0.2 \mathrm{~mm}$ and $1.0 \mathrm{~mm}$.

Use $0.2 \mathrm{~mm}$ displacement cam for high gain (HG) vertical and horizontals, as well as standard gain (SG) horizontals. Use $1.0 \mathrm{~mm}$ displacement cam for SG vertical.

To change cam, place the hex driver through the table platform holes indicated by blue arrows (below left). Loosen hex screws.

Lift brass rod located above cams which are indicated by blue arrow (below right) and slide cam assembly all the way to "stop". Cam assembly moves in the direction of red arrows (below right).
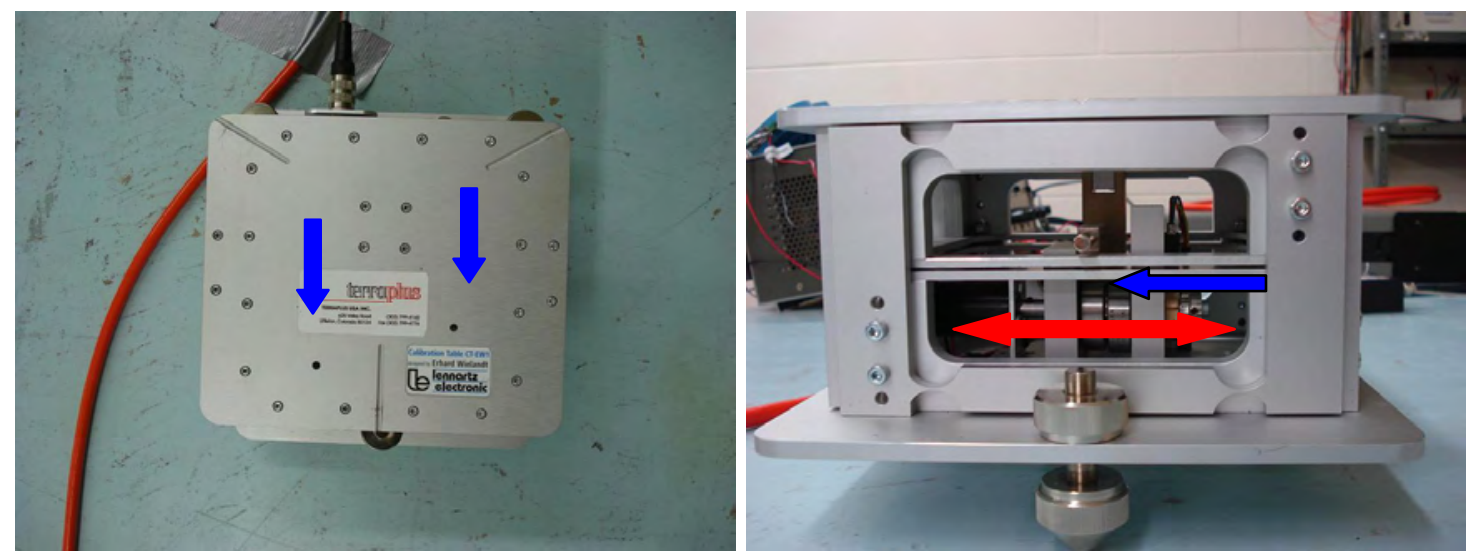

With proper cam selected, tighten hex screws.

Next: connect step table wiring.

a) Connect step table to actuator box using supplied cable. Step table end is a cannon plug.

b) Connect Bayonet Neill-Concelman (BNC) connector on other side of actuator box to the BNC signal output connector on function generator.

c) Connect positive and negative wires from step table to DC power supply (should be set to 9 VDC).

Center step table leveling bubble (below), using screw-adjustable feet. 


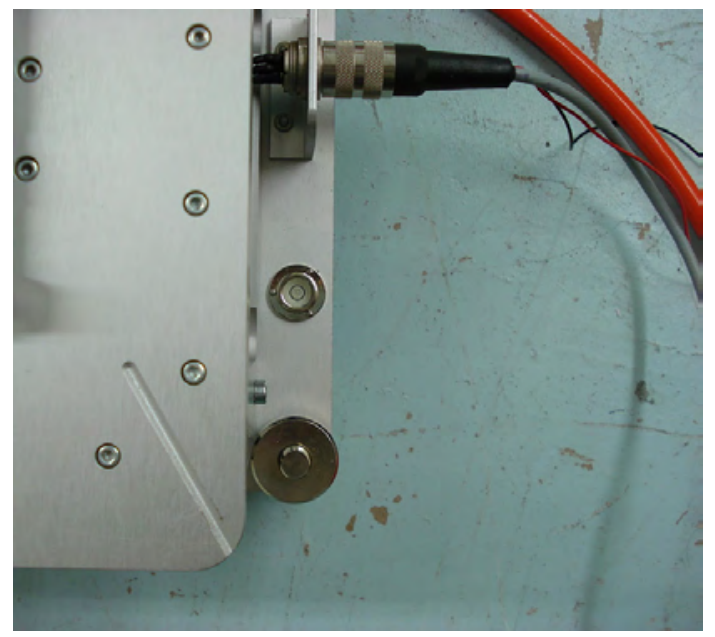

When level, tighten lock knobs.

\section{Part B: Sensor placement, leveling, and electronic centering}

\section{i. Vertical Component}

1. Place sensor directly on top of step table with feet in grooves. Orientation is not important for the vertical calibration.

2. Center leveling bubble on STS-2 using screw adjustable feet.

3. Connect STS-2 cables to instrument, host box, and digitizer. Ensure that cable is free from touching the step table or anything else that could cause interference of motion.

4. Secure cable to pier with tape.

5. When level, tighten lock knobs and unlock masses using screwdriver in slots above each foot.

6. At host box, check mass positions with volt meter using $\mathrm{T}$, $\mathrm{U}$, and $\mathrm{V}$ conductors to $\mathrm{F}$ conductor for signal common.

a) If all positions read less than \pm 5.0 volts, instrument is sufficiently centered.

b) If one or more readings are greater than \pm 5.0 volts, press centering button on host box and check again. Repeat until mass positions between -5.0 and +5.0 volts are achieved on all three masses.

\section{Proceed to Section II to test vertical component, or set up for testing horizon- tal components as follows:}

\section{ii. Horizontal Components}

1. Calibration of the horizontal components requires the use of additional step table equipment: the bearing arm and the tilt table. The tilt table consists of three pieces, the bearing arm consists of one. 


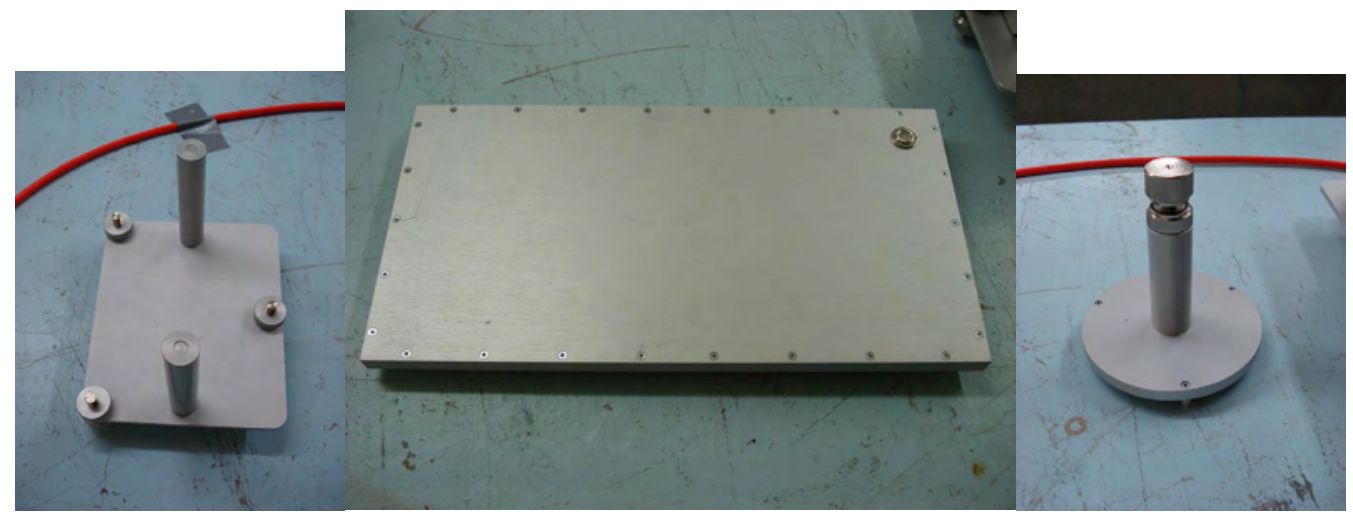

Top: tilt table and bases

Bottom: bearing arm

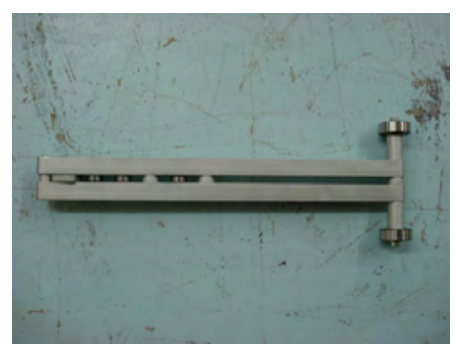

Assembly should look like this when together correctly.

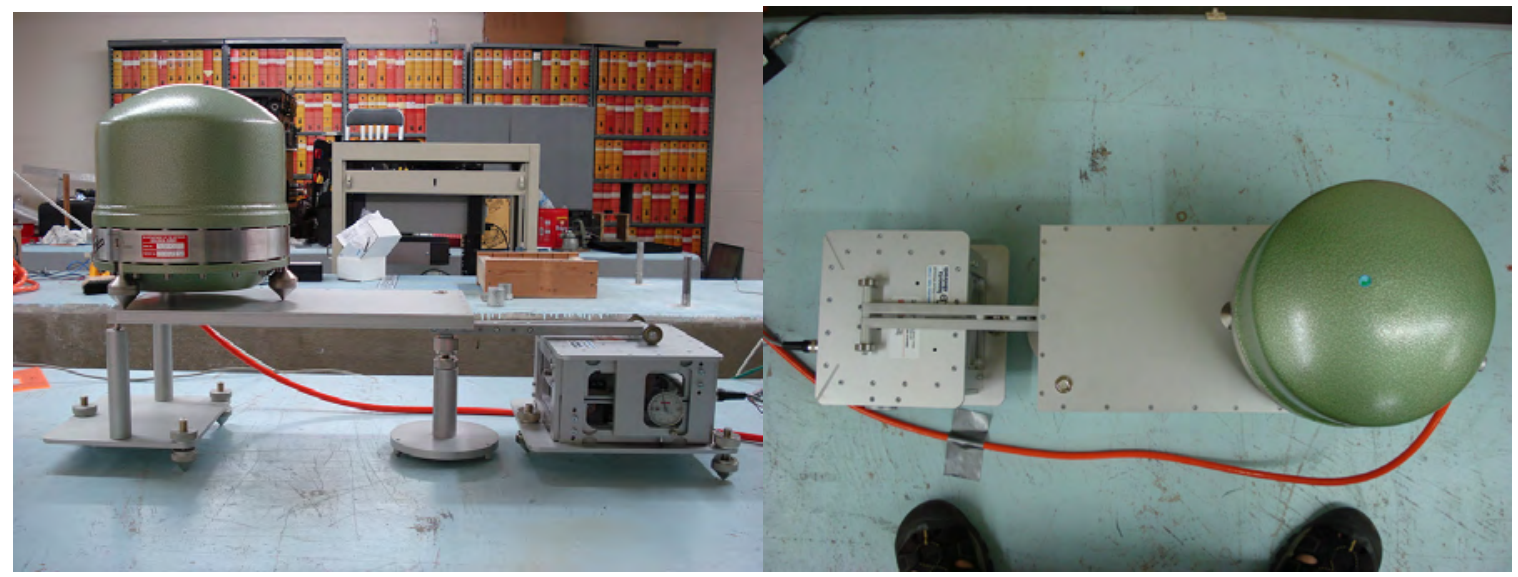

Make sure of the following:

a) "Wheels" on end of bearing arm should be centered on step table directly above and between the two dial gages.

b) The tilt table has a foot that is designed to sit on the bearings between the rails of the bearing arm. This foot should sit on the bearing furthest from the "wheels" for HG seismometer, and bearing closest to the "wheels" for SG seismometer

c) The other feet of the platform sit inside indentions on top of the square base piece.

d) Ensure that the tilt table and bearing arm are aligned with the step table (diagram below) 


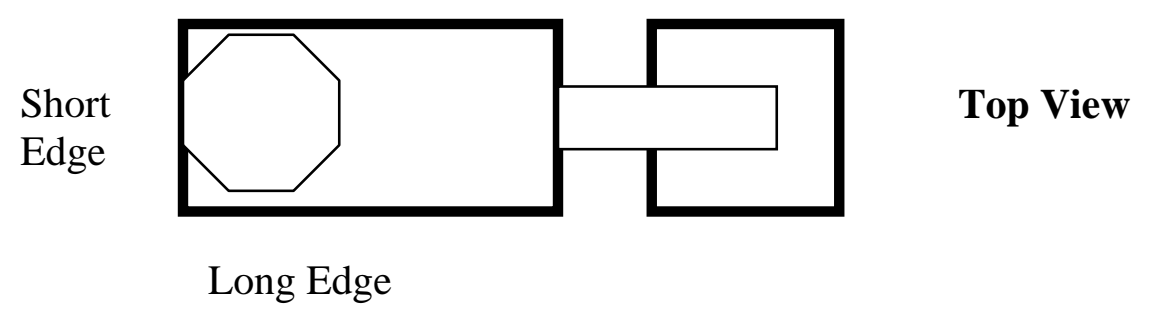

2. Place the sensor on the tilt table and orient. The component to be calibrated should be parallel to the long edge of the tilt table.

Orientation is done for east-west by using a straight edge to space two of the seismometer feet equally from the short edge.

Orientation is done for north-south by using a straight edge to space those same feet equally from the long edge.

In either case, the sensor should be at the end of the tilt table, as far away from the step table as possible

3. Center the leveling bubble on the tilt table

4. Center leveling bubble on STS-2

5. Connect STS-2 cables to instrument, host box, and digitizer. Ensure that cable is free from touching the step table or anything else that could cause interference of motion.

6. Secure cable to pier with tape.

7. When level, tighten lock knobs on feet and unlock masses using screwdriver in slots above each foot.

8. Check mass positions with volt meter using $\mathrm{T}, \mathrm{U}$, and $\mathrm{V}$ conductors on host box.

a) If all positions read less than \pm 5.0 volts, instrument is sufficiently centered

b) If one or more readings are greater than \pm 5.0 volts, press centering button on host box and check again. Repeat until mass positions of less than 5.0 volts or more than -5.0 volts are achieved on all three masses.

\section{Proceed to Section II to test horizontal components.}




\section{Function configuration and running of sequence}

\section{Part A: Configuring DB 360 Function Generator}

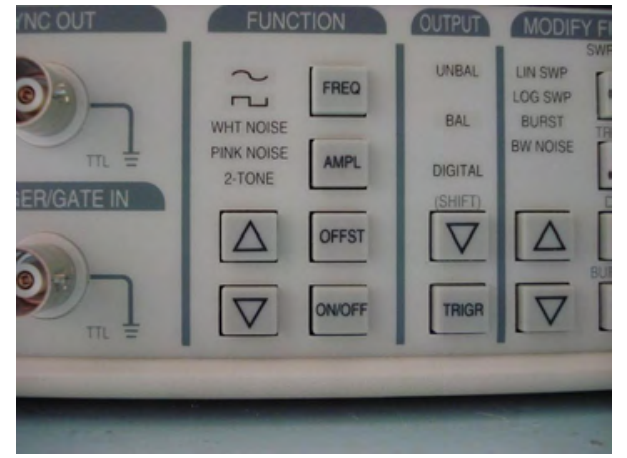

1) Power on function generator (bottom right of DB360 face).

2) Press button for square wave.

3) Frequency should be set to $0.1 \mathrm{~Hz}$ for horizontals and $0.05 \mathrm{~Hz}$ for verticals.

4) Set amplitude to 10 Volts peak-to-peak.

5) Set offset to 5 Volts.

\section{Part B: Measuring $\Delta \mathbf{P}$}

1. $\Delta \mathrm{P}$ refers to change in position of the step table. This is a vertical displacement and is measured by two dial gages, one on each side of the step table.

2. After a sensor is installed on top of step table, $\Delta \mathrm{P}$ will remain constant for calibration sequence.

3. On the actuator box, switch the toggle switch labeled with OSC/Button to "Button".

4. Ensure that "output on/off” button on function generator has output off.

5. When the actuator button is pressed, the step table will move approximately $0.2 \mathrm{~mm}$ or $1.0 \mathrm{~mm}$ depending on which cam is in use.

6. Obtain a value for actual displacement of the step table by taking the difference of dial gage values before and after actuator button is pressed.

Repeat this for micrometer two on the other side of the step table.

Note: The dial gage displacement must be read immediately before running the sequence for vertical and horizontal components. Displacement is effected by the amount of weight on the step table, so the seismometer should be in place.

The two $\Delta \mathrm{P}$ values for each sequence should be very close and will be averaged to get a $\Delta \mathrm{P}$ representative of the whole platform. 


\section{Part C: Running sequence}

1. Cover the entire step table/sensor assembly with a foam box, again keeping cables clear of contact.

2. Switch actuator toggle to "OSC" for oscillator.

3. Press "output on/off" button on function generator to turn drive on.

4. Record time in GMT.

5. Note: it is a good idea to check the data feed with Willard during the first sequence to ensure data is being transferred properly.

6. Allow step table to be driven for 10 min of logging.

7. Press "output on/off" button on function generator to turn drive off.

8. Record time in GMT.

\section{Processing of step table data}

Part 1: Data acquisition and conversion

1. Go to baler web page through Q330

2. Create a folder under $\mathrm{N}:$ ISensOrLoclSensors for the sensor tested using serial number for the name

3. Download data file for test periods with request starting $2-3 \mathrm{~min}$ before and ending $2-3 \mathrm{~min}$ after to the folder just created for your sensor (seed name is BH1 or BH2).

4. Import data file to Cimarron program for plotting.

5. Under file menu, click "index mini seed files" and select your downloaded files.

6. Plot the data.

7. Zoom in on data where peak to peak becomes symmetrical. Cut out data at left and right if necessary using lines dragged from the edges and zoom button at top.

8. Click ASL at top of Cimarron dialog to generate ASL output (this is an ASCII file).

9. Save a copy of this in N:[?]/SensOrLoc/Reference systems/software and also in the sensor folder created above N:ISensOrLoclSensors\\#\#\#\#\#.

10. Repeat the above for all three components (east-west, north-south, Z).

Part 2: Running the analysis

1. Go to N:/SensOrLoc/Reference systems/software.

\section{Vertical Component}

2. Open dispcal.par parameter file.

3. Correct line one for HG or SG, date, and your name.

4. Correct line two for the name of the ASCII file generated in part 1.

5. Correct line three for Serial number and component letter (x, y, or z).

6. Correct line seven with the average $\Delta \mathrm{P}$ value from test.

7. Save and close. 
8. In $\mathrm{N}$ :/SensOrLoc/Reference systems/software find dispcal application file, double click to run.

9. This will generate a dispcal output file in the format: xx_AZI2_06131443.out. Find and open this file.

10. Locate raw generator constant, should be within 2 percent of the manufacturer specification.

11. Save this file in your sensor folder in the format: 008_04_10_TST3_STS2_30748_Z_out.

12. At this point there should be three files saved in that SensOrLoc station file: the seed file, the ASCII file, and your output.

\section{Horizontal Component}

1. Open tiltcal.par parameter file.

2. Correct line one for the gain of the sensor being tested, date, and your name.

3. Correct line two for the name of the ASCII file generated in part 1.

4. Correct line three for Serial number and component letter (x, y, or z).

5. Correct line seven with the result of the following formula: $[(9.79188) \times(\operatorname{avg} . \Delta \mathrm{P})] / 396 \times 10^{-3}$ $=$ equivalent horizontal acceleration due to tilt.

6. Enter to line seven the result of: EHA/3 for standard gain.

7. EHA/12 for high gain.

8. Save and close.

9. In N:/SensOrLoc/Reference systems/software find tiltcal application file, double click to run.

10. This will generate a tiltcal output file. Find and open this file.

11. Locate raw generator constant, should be within 2 percent of the manufacturer specification.

12. Save this file in the format of: 2008_04_10_TST3_STS2_30748_Z_out in the appropriate file under SensOrLoc for the station the sensor belongs to.

13. Repeat for other horizontal component, and make sure all 3 files for each component are saved. Your sensor folder should have a total of 9 files at the end of this. 


\section{Appendix 2: Sample Test Procedure for WM BB Velocity Sensor Frequency Response}

\section{KS54000i Test Procedure (Data Channel Fre- quency Response)}

Purpose of Test: To check the data channel frequency response from $0.001 \mathrm{~Hz}$ to $20 \mathrm{~Hz}$.

Test Location Requirements: The seismometer will be installed and leveled in the ANMX test hole.

Test Equipment Required: Spectrum Analyzer.

Procedure: The frequency response will be run for each data channel. The frequency range will be $0.001-20 \mathrm{~Hz}$. The frequency response will be run in the capacitive mode.

1. Disconnect the cable between the wellhead terminal and the Quanterra test system.

2. Connect the test setup as shown in the second photograph for the vertical channel. Set up the spectrum analyzer as follows:
a. Measure Mode
Swept Sine
Log Sweep
A Gain On (Auto gain)
A Gain Select
Ref CHAN 2
Ref Level 5V
Source Limit 5V
FREQ START Freq $0.001 \mathrm{~Hz}$
STOP Freq $20 \mathrm{~Hz}$
RESLTN AU (Resolution Auto)
AVG Number of Averages 10
FIXED INTEGRATION
SOURCE SWEEP DOWN

3. Push the Start button and after a few minutes you will see the frequency response curve display working from the highest frequency down (right to left on the screen).

4. After the response gets into the flat portion of the curve turn A GAIN OFF, RESLTN FIXED at about 8 points per decade, and set Number of averages to 1 . This method gives you good coherence at the high frequencies but does not take more than two hours to get the long period response. You can check coherence to see that you it is 1 at the high frequencies.

5. As the curve gets into the lower frequencies, it will update much more slowly-perhaps less than twice an hour.

6. When the measurement has been completed and you have the whole response curve, CURVE FIT the data collected from each component and plot it with the curve fit overla- 
id on the response curve. Edit the poles and zeros and plot the table on the reverse side of the plot. Specify 5 poles and 2 zeros for the CURVE FIT program. Also in using the CURVE FIT program set the FIT FUNCTION to USER WEIGHT and EDIT weight to be 1 over the whole pass band. You can also use the SYNTH FUNCTION to synthesize a response. A great deal of difference using the user weight fit function may not be noticeable. Note: You may find the curve fit program will find an erroneous high frequency pole and not find the $-719.507 \mu \mathrm{Hz}$ pole. Delete the erroneous pole and enter the $719.507 \mu \mathrm{Hz}$ pole. Run the curve fit again with these values and see if the results improve. You can change the values in the poles and rerun to get the best results, but tests have shown that small changes in the poles and zeros have little if any effect on the data. The poles and zeros listed in the manual and also used in the Dataless file are listed below:

$\begin{array}{ll}\text { Poles } & \text { Zeros } \\ -9.4587884 \mathrm{e} 00 & 0.0 \\ -3.6147454 \mathrm{e} 00 & 0.0 \\ \pm 4.3141396 \mathrm{e} 00 & \\ -7.19507 \mathrm{e}-04 & \\ -1.239524 \mathrm{e}-02 & \end{array}$

When the test data has been collected, annotate the data channel frequency calibration worksheet.

Repeat the above steps for the north and east channels. 


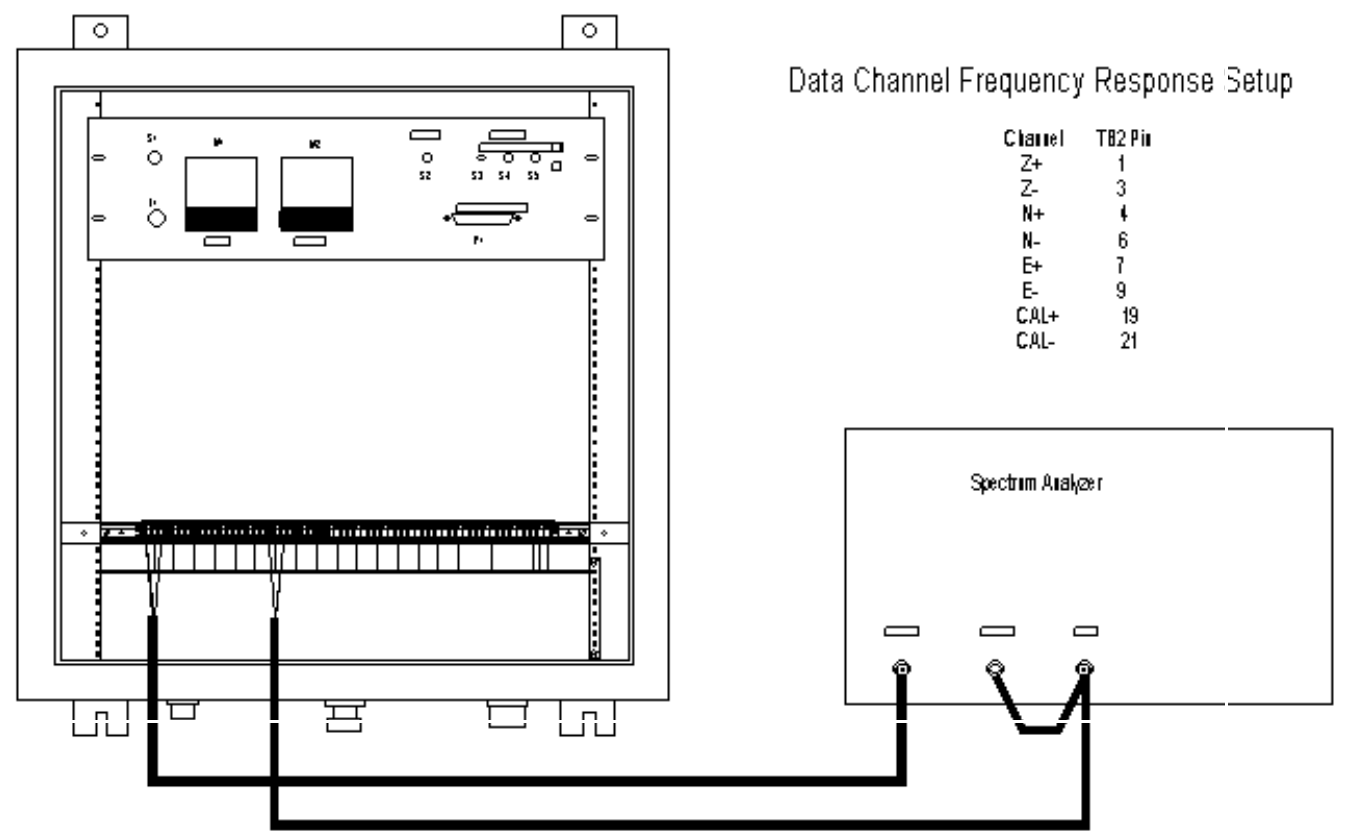

Figure 2. 